\title{
Directed Disulfide Pairing and Folding of Peptides for the De Novo Development of Multicyclic Peptide Libraries
}

Shuaimin $\mathrm{Lu}^{+}$, Yapei $\mathrm{Wu}^{+}$, Jinjing Li, Xiaoting Meng, Chenliang Hu, Yibing Zhao, and Chuanliu $\mathrm{Wu}^{*}$

Department of Chemistry, College of Chemistry and Chemical Engineering, The MOE Key Laboratory of Spectrochemical Analysis and Instrumentation, Xiamen University, Xiamen, 361005, P.R. China.

${ }^{+}$These authors contributed equally to this work.

*Corresponding Author; Email: chlwu@xmu.edu.cn 


\section{Table of Contents}

\section{Experimental section}

1.1 Materials and instruments

1.2 Oxidative folding of peptides

1.2.1 Oxidative folding of peptides containing a $\mathrm{CP}_{n} \mathrm{C}$ motif

1.2.2 Oxidative folding of peptides with formats CPPC-(nAA)-CPPC and CKC-(nAA)-CKC

1.2.3 Oxidative folding of peptides containing two CPPC motifs and two cysteines

1.3 Analysis of disulfide pairing in peptides through orthogonal protecting group strategies

1.4 Analysis of disulfide pairing in peptides by the tryptic digestion strategy

1.5 Scheme S1 | Library construction

1.6 Phage panning

1.7 Fluorescence polarization (FP) competition assay

\section{Results}

2.1 Synthesize the parallel and antiparallel CPPC dimer (Fig S1)

2.2 Mass spectra of the products formed after the oxidation of peptides 1-1、1-2 and 1-3 (Fig S2)

2.3 Chromatograms of the products formed after the oxidation of peptides 2-6 (Fig S3)

2.4 Chromatograms of the products formed after the oxidation of peptides S7-S11 (Fig S4)

2.5 Analysis of disulfide pairing of 7 (Fig S5)

2.6 Analysis of disulfide pairing of 8 (Fig S6)

2.7 Analysis of disulfide pairing of 9 (Fig S7)

2.8 Analysis of disulfide pairing of $\mathbf{1 0}$ (Fig S8)

2.9 Analysis of disulfide pairing of $\mathbf{1 1}$ (Fig S9)

2.10 Alignment of 60 clones' sequences from the library (Fig S10)

2.11 Alignment of the selected sequences (Fig S11)

2.12 Analysis of disulfide pairing of $\mathbf{1 2}$ (Fig S12)

2.13 Binding affinity of the oxidized peptide S16 to MDM2 (Fig S13)

2.14 Analysis of disulfide pairing of $\mathbf{1 3}$ (Fig S14)

2.15 FP competition curves of the binding of peptides (oxidized 12 and 13) to MDM2 (Fig S15) 


\section{Experimental section}

\subsection{Materials and instruments}

All the Fmoc-protected amino acids, Rink amide MBHA resin and Wang resin used for peptide syntheses were supplied by GL Biochem (Shanghai, China). Acetonitrile (ACN), trifluoroacetic acid (TFA), glutathione reduced (GSH), glutathione oxidized (GSSG), dithiothreitol (DTT), $\mathrm{NaH}_{2} \mathrm{PO}_{4} \cdot 2 \mathrm{H}_{2} \mathrm{O}, \quad \mathrm{Na}_{2} \mathrm{HPO}_{4} \cdot 12 \mathrm{H}_{2} \mathrm{O}$ and meta-phosphoric acid $\left(\mathrm{HPO}_{3}\right)$ were purchased from Sigma-Aldrich (Beijing). Tris (2-carboxyethyl) phosphine hydrochloride (TCEP) was bought from Energy Chemical (Shanghai, China). All the chemicals used to prepare the 1xTBE (Tris-Cl, $\mathrm{Na}_{2}$ EDTA $2 \mathrm{H}_{2} \mathrm{O}$, boric acid), $3 \mathrm{M} \mathrm{NaAc}\left(\mathrm{NaAc} \cdot 3 \mathrm{H}_{2} \mathrm{O}, \mathrm{pH} 5.2\right), 1 \times \mathrm{TE}(10 \mathrm{mM}$ Tris-HCl, $1 \mathrm{mM}$ EDTA $\left.\cdot \mathrm{Na}_{2} \cdot 2 \mathrm{H}_{2} \mathrm{O}, \mathrm{pH} 8.0\right), 100 \mathrm{mM} \mathrm{NaAc}\left(\mathrm{NaAc} \cdot 3 \mathrm{H}_{2} \mathrm{O}, \mathrm{pH} 4.5\right), 1 \mathrm{mM}$ EDTA (EDTA $\cdot \mathrm{Na}_{2} \cdot 2 \mathrm{H}_{2} \mathrm{O}$, $\mathrm{pH}$ 8.0), $0.1 \%$ SDS, binding buffer (10 mM Tris-Cl, $150 \mathrm{mM} \mathrm{NaCl}, 10 \mathrm{mM} \mathrm{MgCl} 2,1 \mathrm{mM} \mathrm{CaCl}_{2}$, pH 7.4), washing buffer (binding buffer containing $0.1 \%$ Tween-20), blocking buffer (binding buffer containing $0.3 \%$ Tween-20 and $3 \%$ (w/v) BSA), elution buffer (50 mM glycine, pH 2.2), neutralization buffer (1 M Tris-Cl, pH 8.0), PEG/NaCl (20\% PEG-6000 (w/v), $2.5 \mathrm{M} \mathrm{NaCl})$ and 2YT medium (16 g tryptone, $10 \mathrm{~g}$ yeast extract, $5 \mathrm{~g} \mathrm{NaCl}$ per $1 \mathrm{~L} \mathrm{dH}_{2} \mathrm{O}$ ) were purchased from Sigma-Aldrich (Beijing, China), Sangon Biotech (Shanghai, China) or Sinopharm Chemical Reagent (Beijing, China).

All peptides were synthesized on a CEM Discover Liberty BLUE microwave-assisted peptide synthesizer. High performance liquid chromatography (HPLC) performed by a SHIMADZU system equipped with a quaternary solvent manager (QSM), a sample manager with flow-through needle (SM-FTN), a column manager, and an AQUITY ® PDA detector was used for the purification and analysis of peptides. A HITACHI U-3900H UV/Vis spectrometer was used for the quantification of peptides. A Bruker Esquire 3000 plus ion trap ESI mass spectrometry (MS) and Autoflex maX MALDI-TOF Ms were applied to identify the formed peptides. Fluorescence polarization was recorded using a Tecan Infinite® 200 PRO Microplate Reader.

\subsection{Oxidative folding of peptides}




\subsubsection{Oxidative folding of peptides containing a $\mathrm{CP}_{n} \mathrm{C}$ motif}

The fully reduced peptides $(200 \mu \mathrm{M})$ were dissolved in $100 \mathrm{mM}$ phosphate buffer $(\mathrm{pH} 7.4)$ containing $~ 30$ vol\% DMSO at room temperature for $\sim 10 \mathrm{~h} .{ }^{1}$ After that, aliquots were immediately transferred to a tube containing $10 \mathrm{wt} \% \mathrm{HPO}_{3}$ to quench the reactions. The peptides were then analyzed by the analytical HPLC.

\subsubsection{Oxidative folding of peptides with formats CPPC-(nAA)-CPPC and CKC-(nAA)-CKC}

Briefly, $50 \mu \mathrm{M}$ peptide and $0.5 \mathrm{mM}$ GSSG were co-incubated in $100 \mathrm{mM}$ phosphate buffer $(\mathrm{pH}$ 7.4). After the reaction for $4 \mathrm{~h}$ at room temperature, the reaction was quenched with $10 \mathrm{wt} \% \mathrm{HPO}_{3}$ and then the sample was analyzed by HPLC. ${ }^{2}$

\subsubsection{Oxidative folding of peptides containing two CPPC motifs and two cysteines}

To an empty tube, the fully reduced peptides $(50 \mu \mathrm{M})$ dissolved in $100 \mathrm{mM}$ phosphate buffer (pH 7.4) containing $0.5 \mathrm{mM}$ oxidized glutathione (GSSG) were added. Four hours later, aliquots were quenched with $10 \%$ aqueous $\mathrm{HPO}_{3}$, and then monitored using HPLC.

\subsection{Analysis of disulfide pairing in peptides through orthogonal protecting group strategies}

In this work, the disulfide pairing in cyclic peptides was determined by the orthogonal protecting group strategy. Firstly, two cysteines of the peptide were replaced with the Acm-protected cysteines. Then the peptide was dissolved in $100 \mathrm{mM}$ phosphate buffer ( $\mathrm{pH} 7.4$ ) containing $0.5 \mathrm{mM}$ GSSG. The oxidation product was purified with HPLC, and recovered by lyophilization. The peptide was reconstituted in an appropriate amount of pure water to achieve a definite concentration. Subsequently, the oxidation product was dissolved with methanol (containing $1 \%$ TFA) to achieve a concentration of $50 \mu \mathrm{M}$, and added dropwise to $2 \mathrm{eq}$ or $10 \mathrm{eq} \mathrm{I}_{2}$ in methanol. ${ }^{3}$ Finally, the oxidation product after deprotection was analyzed by HPLC. Therefore, the disulfide pairing of products directly obtained from oxidative folding can be confirmed by comparing with standard products synthesized through the orthogonal protecting group strategy.

\subsection{Analysis of disulfide pairing in peptides by the tryptic digestion strategy}

To a solution containing the oxidative peptides (isolated by HPLC, $\sim 1-30 \mu \mathrm{g}$ ) in $90 \mu \mathrm{L}$ phosphate buffer $(100 \mathrm{mM}, \mathrm{pH}=6), 10 \mu \mathrm{L}$ aqueous solution of trypsin $(1 \mathrm{mg} / \mathrm{mL})$ was added. The solution was kept at room temperature for $0.5 \mathrm{~h}$. Subsequently, the digested fragments were analyzed 
by LC-MS and/or comparing with the parallel and antiparallel CPPC dimers synthesized through the orthogonal protecting grouping strategy.

\subsection{Scheme S1 | Library construction}

The schematic diagram of construction of the phage-displayed DRP library with a cysteine framework analogous to peptide $\mathbf{9}\left(\mathrm{CPPCX}_{5} \mathrm{CX}_{5} \mathrm{CX}_{5} \mathrm{CPPC} ; \mathrm{X}\right.$ is any amino acid encoded by $\left.\mathrm{NNK}\right)$ was presented in Scheme S1. The procedures were described below. First, a chemosynthetic 123-bp oligonucleotide and an extension primer were co-incubated to achieve annealing (heated to $95{ }^{\circ} \mathrm{C}$ and cooled slowly in ice). After that, it became the double strand through extension $\left(4 \mathrm{~h}, 37^{\circ} \mathrm{C} ; 15\right.$ $\min , 65^{\circ} \mathrm{C}$ ) with Klenow fragment (NEB). Next, the extension product and phage vector were digested with SfiI $\left(12 \mathrm{~h}, 50^{\circ} \mathrm{C}\right)$ and NotI $\left(12 \mathrm{~h}, 37^{\circ} \mathrm{C}\right)$. Subsequently, the double strand primers and phage vector were purified by polyacrylamide gel electrophoresis (PAGE) and agarose gel electrophoresis respectively. Afterward, the purified SfiI/NotI-digested DNA fragment was linked to the digested pCANTAB overnight at $16{ }^{\circ} \mathrm{C}$. The next day, the ligation product was purified and transfected into electrocompetent ER2738 cells with the optimum insert/vector ratio (10/1) by electroporation $(200 \Omega, 2.5 \mathrm{kV})$. Finally, the transfected cells were revived and measured the titer (library size: $1.0 \times 10^{9}$ ). To ensure the accuracy and diversity of the final phage library, 60 phage clones were selected randomly for DNA sequencing.

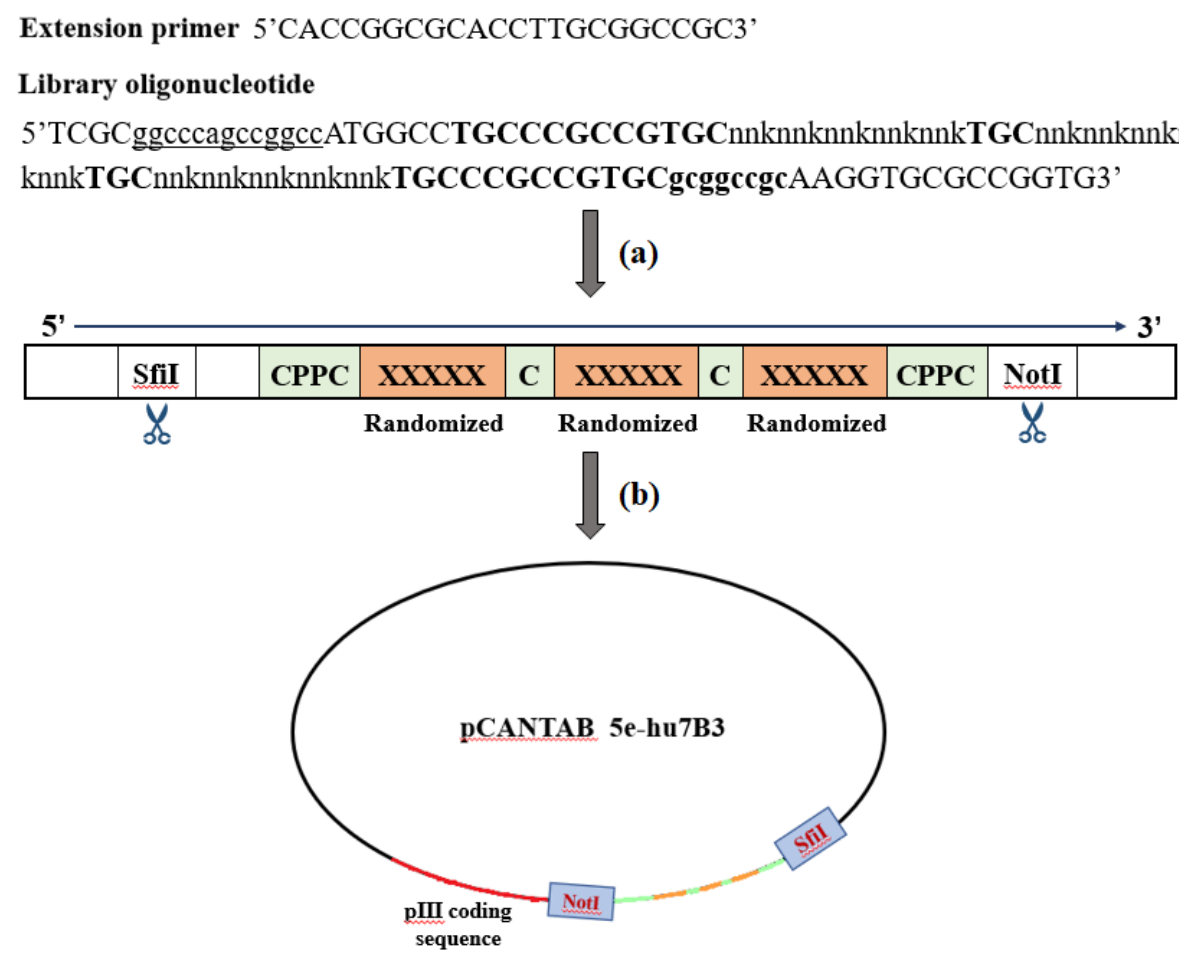


Scheme S1. Schematic presentation of the cloning of randomized inserts into the phage vector; (a) annealing and extension; (b) double digestion, followed by DNA-ligation with pCANTAB in digested form. Nucleotide and the primer sequence used for synthesizing randomized inserts are shown: Cut site of restriction endonuclease SfiI is lowercase and underlined. Cut sites of NotI are lowercase and in bold. The cysteine framework is indicated in bold and uppercase.

\subsection{Phage panning}

Firstly, a monoclone of ER2738 cells was selected from a tetracycline agar plate and cultured in $5 \mathrm{ml} 2 \mathrm{YT}$ medium for subsequent titer determination after screening. $50 \mu \mathrm{L}$ of the streptavidin-coated magnetic beads (or neutravidin-coated magnetic beads for the second-round panning) was placed in two low-adsorption centrifugal tubes. After removal of the supernatant, the magnetic beads were cleaned twice with a $1 \mathrm{~mL}$ binding buffer on the magnet. $10 \mu \mathrm{g}$ of the target protein MDM2 was added to one of the tubes, and the same volume of binding buffer were added to the other. Incubated in a 3D shaker at room temperature for $10 \mathrm{~min}$, then the beads were washed with $1 \mathrm{~mL}$ binding buffer three times. The beads were suspended with $300 \mu \mathrm{L}$ binding buffer, and $150 \mu \mathrm{L}$ blocking buffer was added immediately to incubate for 2 hours at room temperature for blocking. Identically, the phage library (titer $\approx 10^{12}$ ) was blocked with $3 \mathrm{~mL}$ binding buffer and $1.5 \mathrm{~mL}$ blocking buffer. After the blocking, phage library was divided into two equal parts. Magnetic beads of the experimental group (added the target protein MDM2) and control group were mixed with phage library and co-incubated in a $3 \mathrm{D}$ shaker at room temperature for 30 minutes. After the incubation, discarded the supernatant and washed the beads for eight times with washing buffer and with Binding buffer twice. During the cleaning process, the low-adsorption centrifugal tubes should be replaced at least three times to reduce the non-specific adsorption. After the last washing, the magnetic beads were resuscitated with $200 \mu \mathrm{L}$ of elution buffer at room temperature for $10 \mathrm{~min}$. Afterward, the supernatant was transferred to a new centrifuge tube with $40 \mu \mathrm{L}$ neutralization buffer for neutralization. Taking $10 \mu \mathrm{L}$ of the neutralizing eluent of experimental group and control group for the succedent phage titration. After three and four rounds of panning as described above, phage clones were randomly picked from the selection fractions for sequencing.

\subsection{Fluorescence polarization (FP) competition assay}


Firstly, the peptides with the highest enrichment were synthesized using CEM Discover Liberty BLUE microwave-assisted peptide synthesizer and then oxidized them. The oxidation products were purified by HPLC for the subsequent affinity assays with MDM2 using the FP competition assay (Figure S15). All the FP assays were performed in $10 \mathrm{mM}$ PBS on 96-well flat-bottom OptiPlate black plate using a Tecan Infinite ${ }^{\circledR} 200$ PRO Microplate Reader. A fluorescently labeled peptide FITC-PMI (FITC-KGTSFAEYWNLLSP) which has a strong affinity binding to MDM2 was selected as the competing peptide. For the FP competition assay, the fluorescently labeled peptide FITC-PMI was diluted with $10 \mathrm{mM}$ PBS to a final concentration of $20 \mathrm{nM}$. A series of concentrations of peptides $(0-10 \mu \mathrm{M})$ that are gradually diluted and FITC-PMI $(20 \mathrm{nM})$ and SUMO-MDM2 $(80 \mathrm{nM})$ were co-incubated at room temperature for $10 \mathrm{~min}$. Each sample was analyzed three times in parallel. The polarization data were fitted with the equation (1) in Origin 8.5, which is based on one-site competitive model. The inhibition constant $K_{\mathrm{i}}$ values were obtained according to the equation (2). ${ }^{4}$

$$
\begin{gathered}
y=A_{2}+\frac{A_{1}-A_{2}}{1+10^{(x-\log E C 50)}} \\
K_{i}=[I]_{50} /\left([L]_{50} / K_{d}+[P]_{0} / K_{d}+1\right)
\end{gathered}
$$

where $\mathrm{x}$ is the logmolar concentration of the oxidation peptides, $\mathrm{y}$ is the measured fluorescence anisotropy, $A_{1}$ is the top plateaus of anisotropy, $A_{2}$ is the bottom plateaus of anisotropy, $[I]_{50}$ denotes the concentration of the oxidation peptides at $50 \%$ inhibition, $[L]_{50}$ is the concentration of the labeled peptide FITC-PMI at $50 \%$ inhibition, $[P]_{0}$ is the concentration of the free protein at $0 \%$ inhibition, and $K_{d}$ is the dissociation constant of PMI to MDM2. 


\section{Results}

2.1 Synthesize the parallel and antiparallel CPPC dimer through an orthogonal protecting group strategy

(a) 1-K. KGCPPCGW

One cysteine was replaced One cysteine was replaced
with the Acm-protected cysteine
S1. KGC(Acm)PPCGW

DMSO/100 mM PB (pH 7.4)

Oxidized Sl KGC(Acm)PPCGW KGC(Acm)PPCGW

$\varliminf_{2} / \mathrm{CH}_{3} \mathrm{OH}(1 \%$ TFA)

KGCPPCGW

KGCPPCGW
KGCPPCGW (the parallel dimer) $\downarrow$

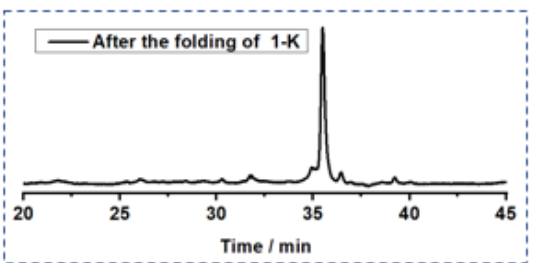

S1. KGC(Acm)PPCGW + Sla. KGCPPC(Acm)GW DMSO/100 mM PB (pH 7.4)

Oxidized S1+Sla KGC(Acm)PPCGW KGCPPC (Acm)GW $\mathrm{I}_{2} / \mathrm{CH}_{3} \mathrm{OH}(1 \% \mathrm{TFA})$ KGCPPCGW WG I (the antiparallel dimer) $\downarrow$
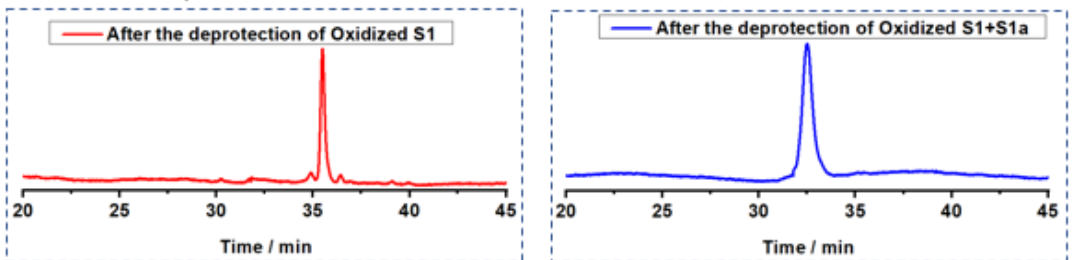

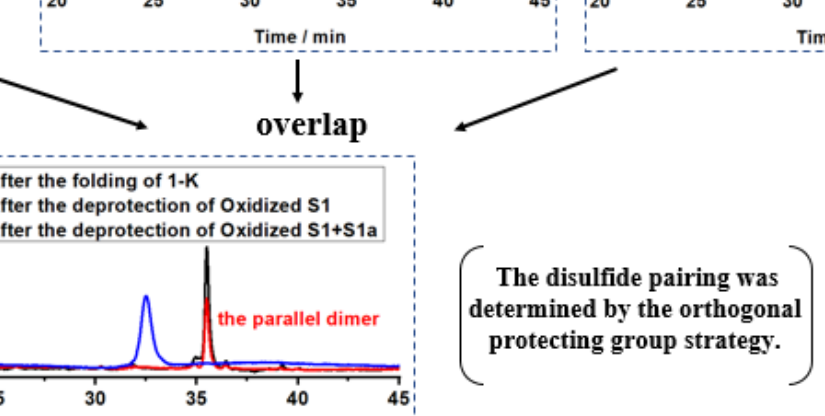



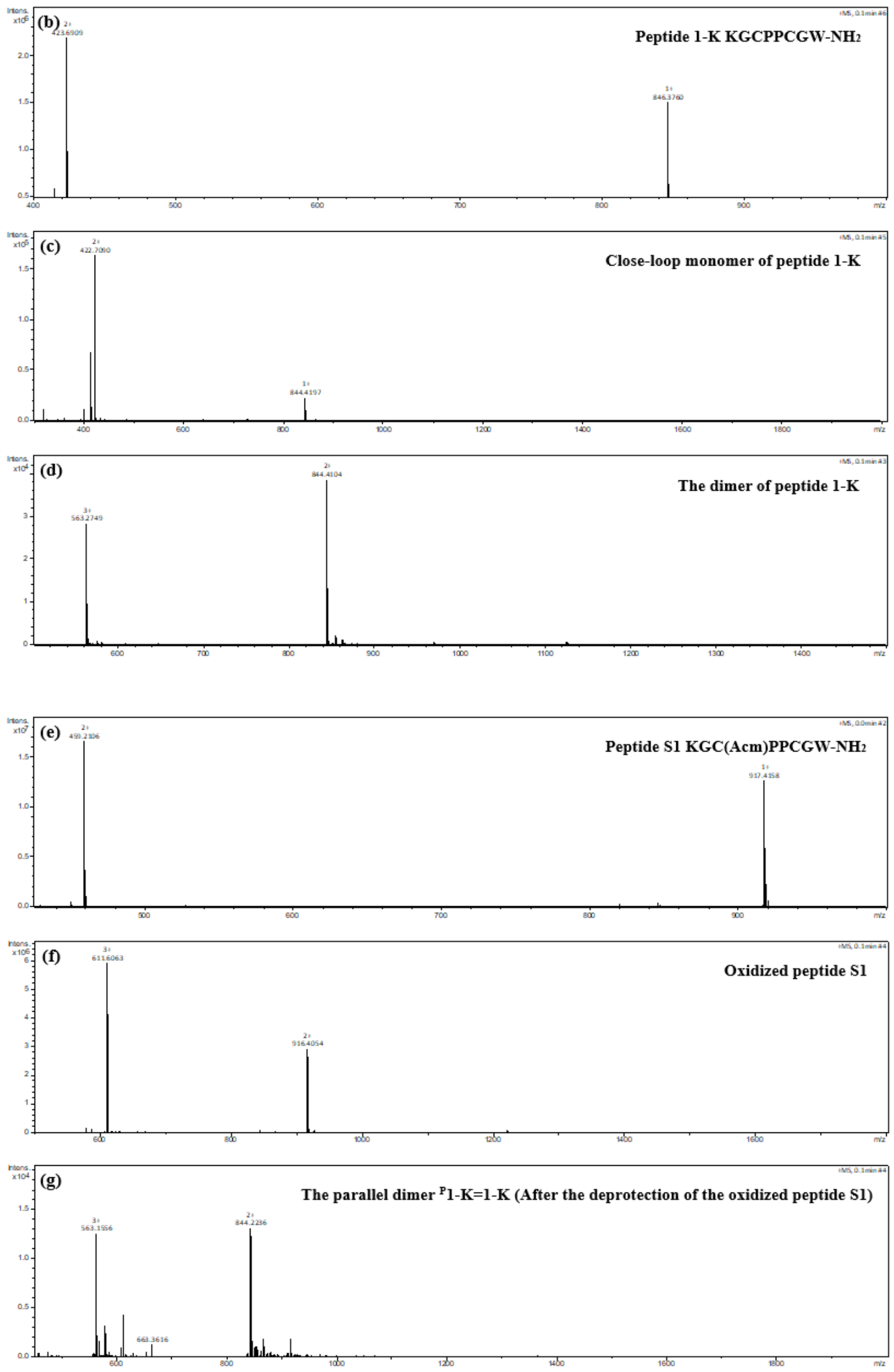

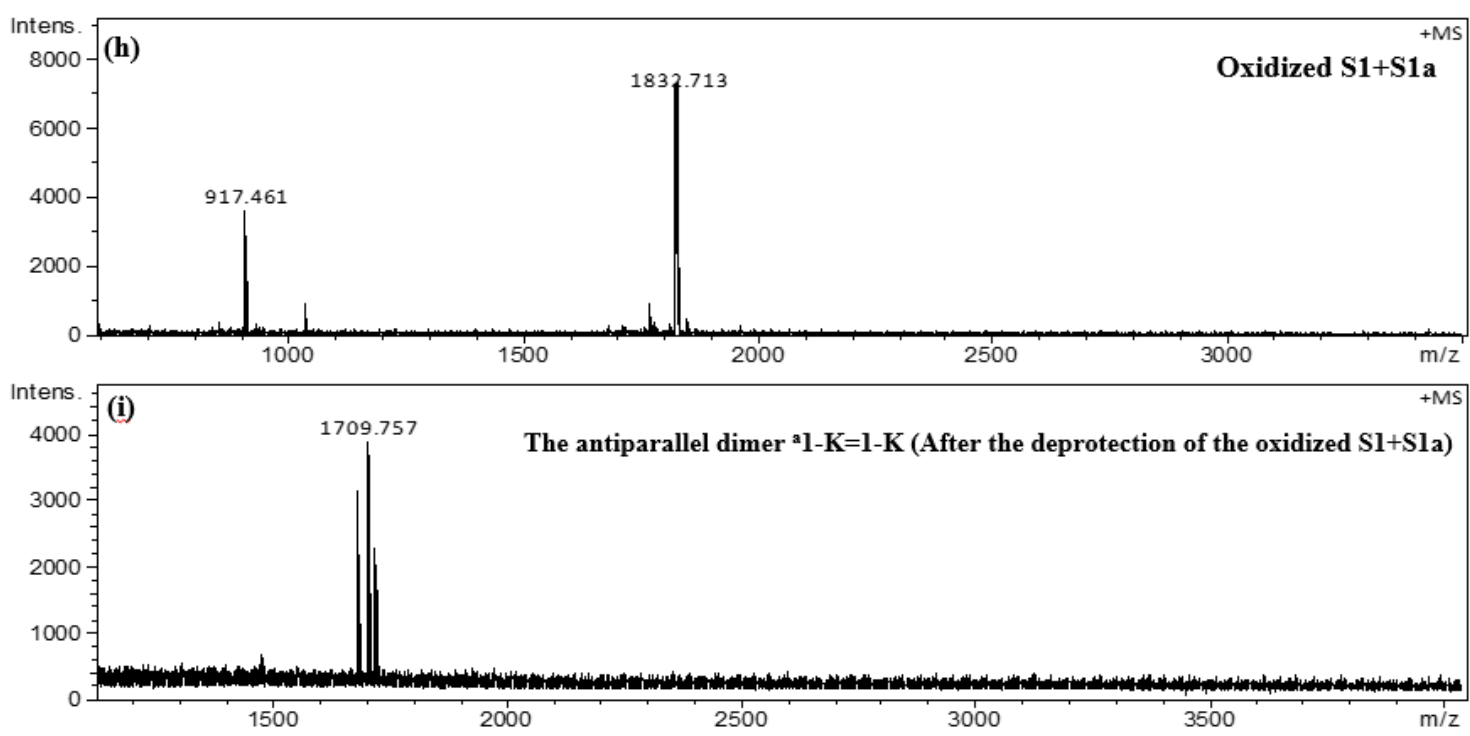

Fig S1. (a) The flowchart to determine the disulfide pairing of 1-K. This result confirms that oxidation of a peptide with a CPPC motif leads to formation of the parallel dimer. (b) Mass spectrum of peptide 1-K. (c) Mass spectrum of close-loop monomer of peptide 1-K. (d) Mass spectrum of the dimer of peptide 1-K. (e) Mass spectrum of peptide S1. (f) Mass spectrum of the oxidized peptide S1. (g) Mass spectrum of the parallel dimer. (h) Mass spectrum of the oxidized S1+S1a. (i) Mass spectrum of the antiparallel dimer. 
2.2 Mass spectra of the products formed after the oxidation of peptides 1-1、1-2 and 1-3
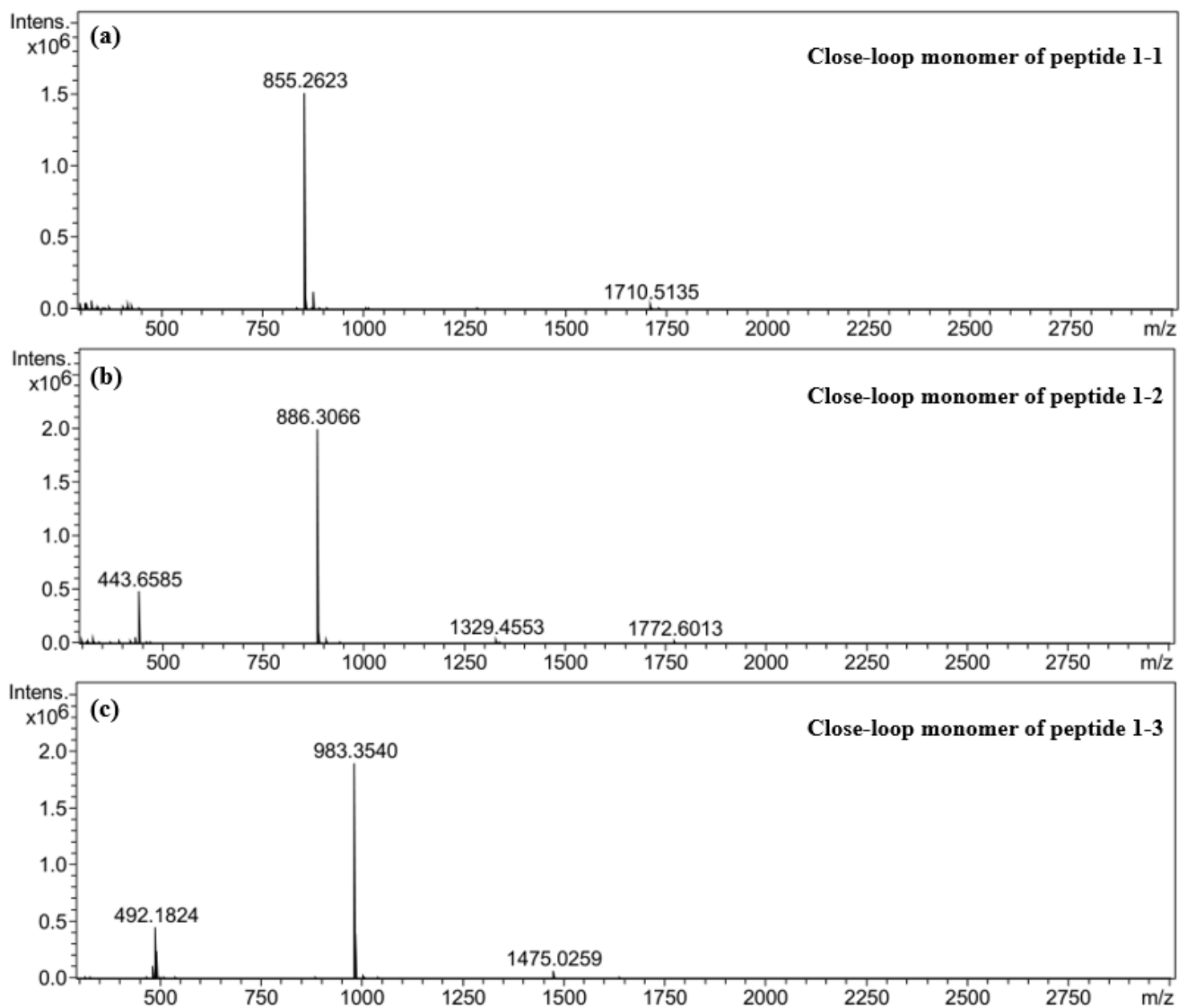

Fig S2. (a) Mass spectrum of close-loop monomer of peptide 1-1 (sequence: GCPPPCGW). (b) Mass spectrum of close-loop monomer of peptide 1-2 (sequence: GCPPKCGW). (c) Mass spectrum of close-loop monomer of peptide 1-3 (sequence: GCPPKCPGW). 


\subsection{Chromatograms of the products formed after the oxidation of peptides 2-6}

(a)

\begin{tabular}{|c|c|c|}
\hline Name & Sequence of shorthand & Sequence \\
\hline Peptide 2 & CPPC-(5AA)-CPPC & H-WGCPPCGGKGGCPPCGW-NH ${ }_{2}$ \\
\hline Peptide 3 & CPPC-(7AA)-CPPC & H-WGCPPCGGGKGGGCPPCG-NH${ }_{2}$ \\
\hline Peptide 4 & CPPC-(9AA)-CPPC & H-WGCPPCGGGGKGGGGCPPCGW-NH ${ }_{2}$ \\
\hline Peptide 5 & CPPC-(13AA)-CPPC & H-WGCPPCGGGGGGKGGGGGGCPPCGW-NH \\
\hline Peptide 6 & CPPC-(18AA)-CPPC & H-WGCPPCSGGKGGSKGGSKGGSKGGCPPCGW-NH \\
\hline Peptide S2 & $\mathrm{CPPC}(\mathrm{Acm})-(5 \mathrm{AA})-\mathrm{C}(\mathrm{Acm}) \mathrm{PPC}$ & H-WGCPPC $(A c m) G G K G G C(A c m) P P C G W-N_{2}$ \\
\hline Peptide S3 & $\mathrm{CPPC}(\mathrm{Acm})-(7 \mathrm{AA})-\mathrm{C}(\mathrm{Acm}) \mathrm{PPC}$ & H-WGCPPC(Acm)GGGKGGGC(Acm)PPCG-NH ${ }_{2}$ \\
\hline Peptide S4 & $\mathrm{CPPC}(\mathrm{Acm})-(9 \mathrm{AA})-\mathrm{C}(\mathrm{Acm}) \mathrm{PPC}$ & H-WGCPPC (Acm)GGGGKGGGGC(Acm)PPCGW-NH ${ }_{2}$ \\
\hline Peptide S5 & $\mathrm{CPPC}(\mathrm{Acm})-(13 \mathrm{AA})-\mathrm{C}(\mathrm{Acm}) \mathrm{PPC}$ & H-WGCPPC(Acm)GGGGGGKGGGGGGC(Acm)PPCGW-NH \\
\hline Peptide S6 & $\mathrm{C}(\mathrm{Acm}) \mathrm{PPC}-(18 \mathrm{AA})-\mathrm{C}(\mathrm{Acm}) \mathrm{PPC}$ & H-WGC(Acm)PPCSGGKGGSKGGSKGGSKGGC(Acm)PPCGW-NH \\
\hline
\end{tabular}

(b) 2. WGCPPCGGKGGCPPCGW (b) 2. WGCPPCGGKGGCPPCGW $\begin{gathered}\text { two cysteines were replaced } \\ \text { with the Acm-protected cysteines }\end{gathered}$

oxidation $0.5 \mathrm{mM}$ GSSG/100 mM PB (pH 7.4)

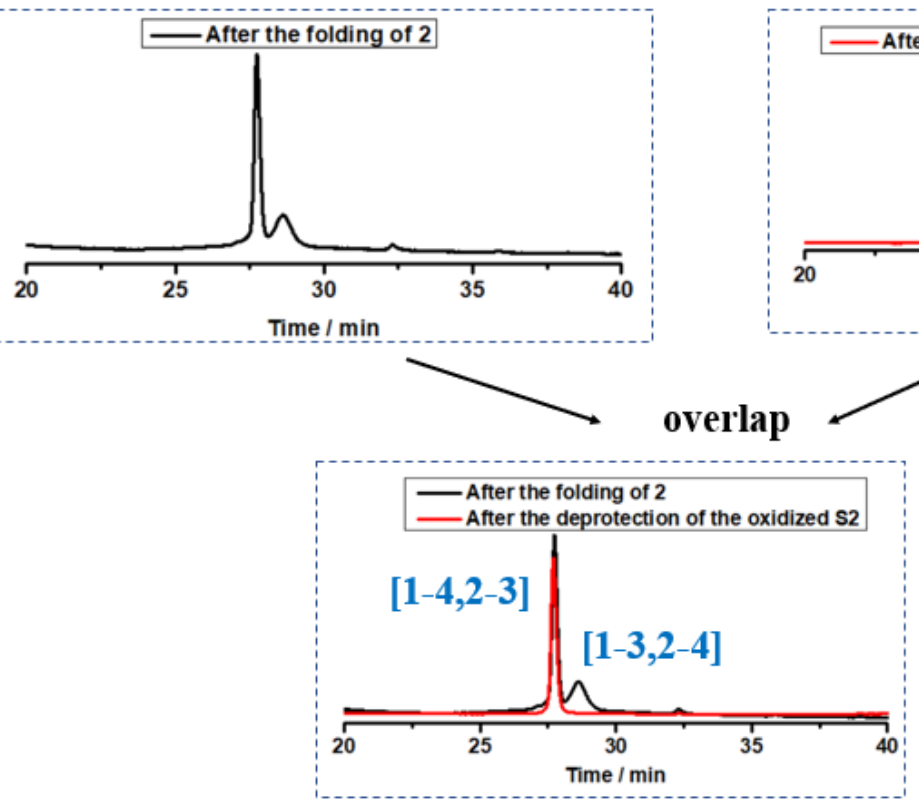

S2. WGCPPC(Acm)GGKGGC(Acm)PPCGW $0.5 \mathrm{mM} \mathrm{GSSG} / 100 \mathrm{mM} \mathrm{PB}(\mathrm{pH} 7.4)$ Oxidized S2 WGCPPC(Acm)GGKGGC(Acm)PPCGW | 2 / СH3OH (1 \%TFA)

WGCPPCGGKGGCPPCGW (disulfide pairing [1-4,2-3]) $\downarrow$

the deprotection of the oxidized S2

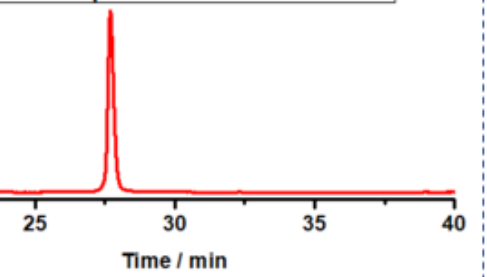

The disulfide pairing was determined by the orthogonal protecting group strategy. 

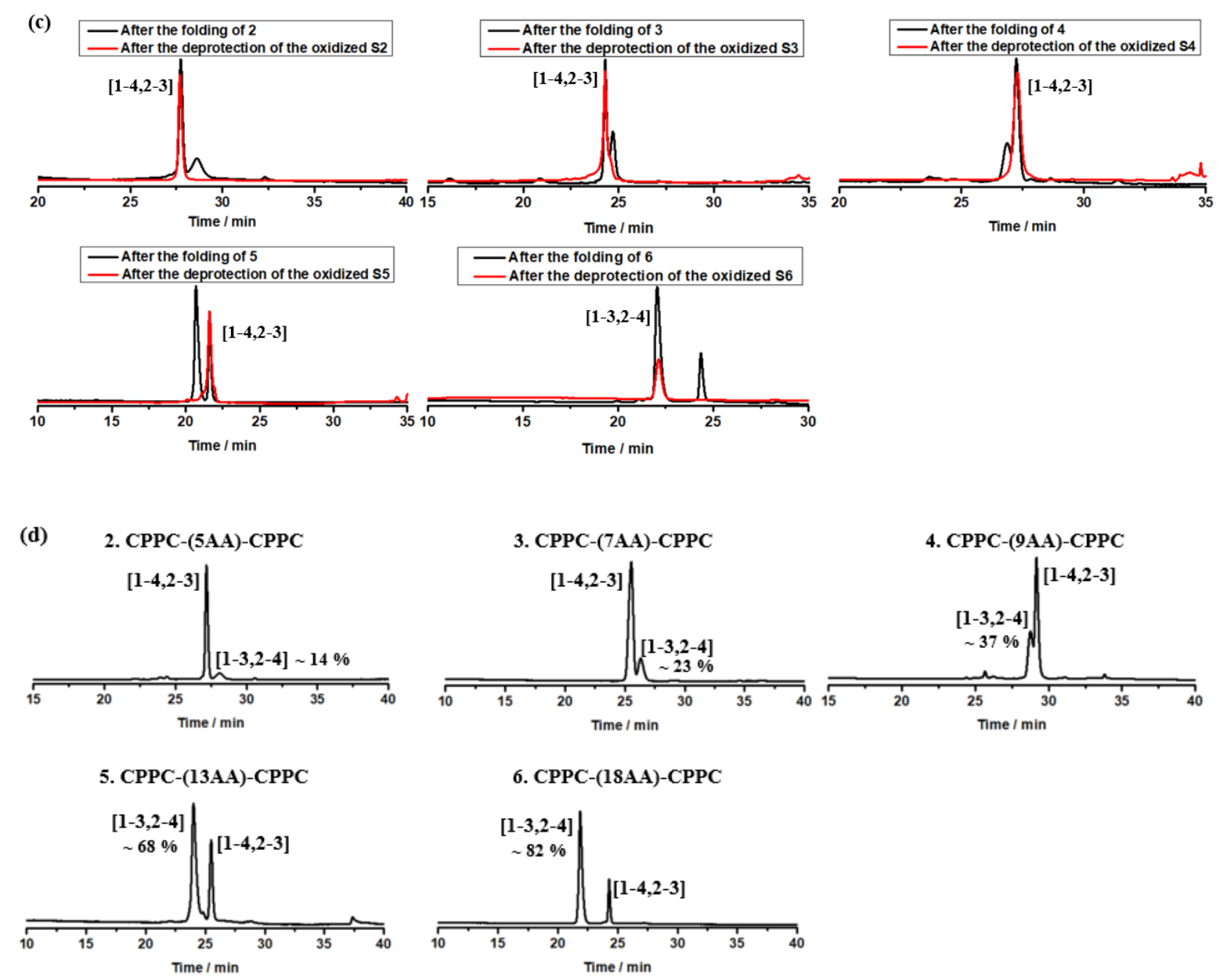

Fig S3. (a) The sequence information of peptides 2-6 and S2-S6. (b) The flowchart to determine the disulfide pairing (take the example of 2). (c) Chromatograms of the products formed from the oxidative folding of $\mathbf{2}, \mathbf{3}, \mathbf{4}, \mathbf{5}$ and $\mathbf{6}$ (black line) and the orthogonal protecting group strategy (red line), respectively. (d) Chromatogram showing the oxidation of 2, 3, 4, 5 and 6 in phosphate buffer (100 mM, pH 7.4) containing $0.5 \mathrm{mM}$ oxidized glutathione (GSSG) to determine the relative yields of the two oxidized products. 
2.4 Chromatograms of the products formed after the oxidation of peptides S7-S11

(a)

\begin{tabular}{ccc}
\hline Name & Sequence of shorthand & Sequence \\
\hline Peptide S7 & CKC-(5AA)-CKC & H-WGCKCGKGGGCKCGW-NH 2 \\
Peptide S8 & CKC-(7AA)-CKC & H-WGCKCGGKGGGGCKCG-NH 2 \\
Peptide S9 & CKC-(9AA)-CKC & H-WGCKCGGGKGGGGGCKCGW-NH 2 \\
Peptide S10 & CKC-(13AA)-CKC & H-WGCKCGGGGGKGGGGGGGCKCGW-NH 2 \\
PeptideS11 & CKC-(18AA)-CKC & H-WGCKCSGGKGGSKGGSKGGSKGGCKCGW-NH 2 \\
\hline
\end{tabular}

(b)
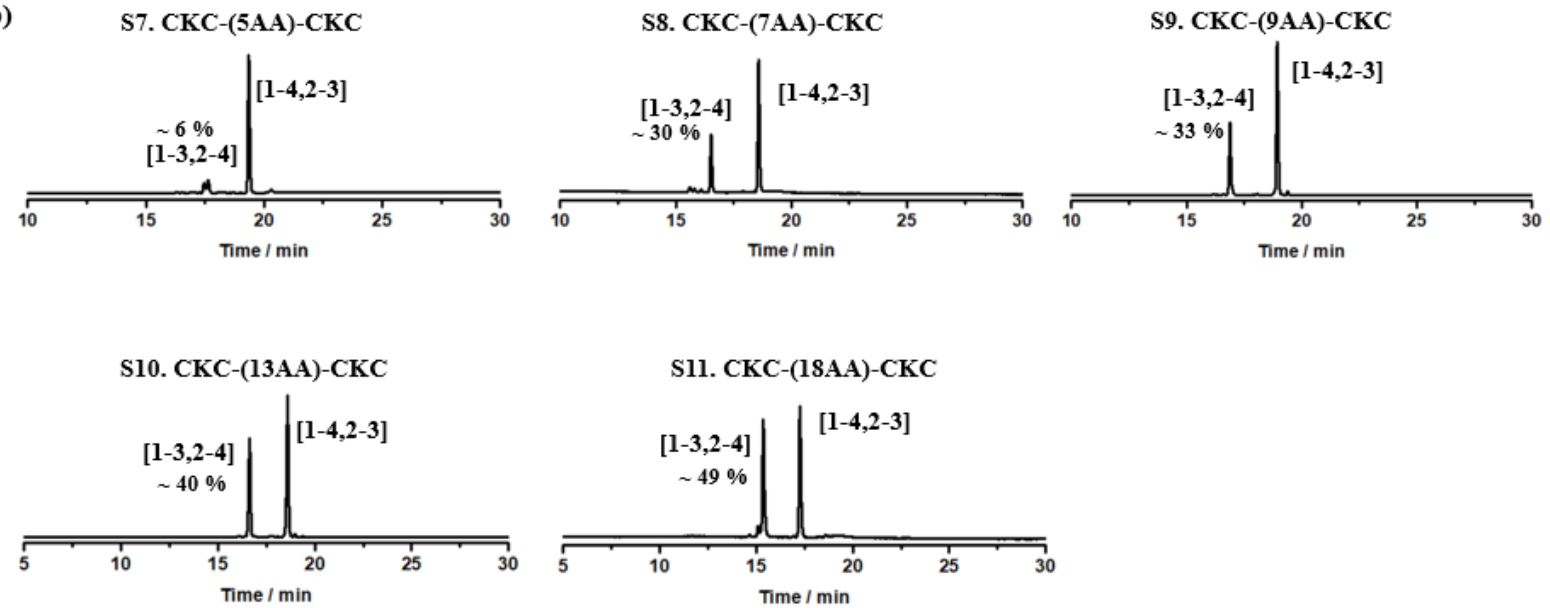
(c) S7. CKC-(5AA)-CKC
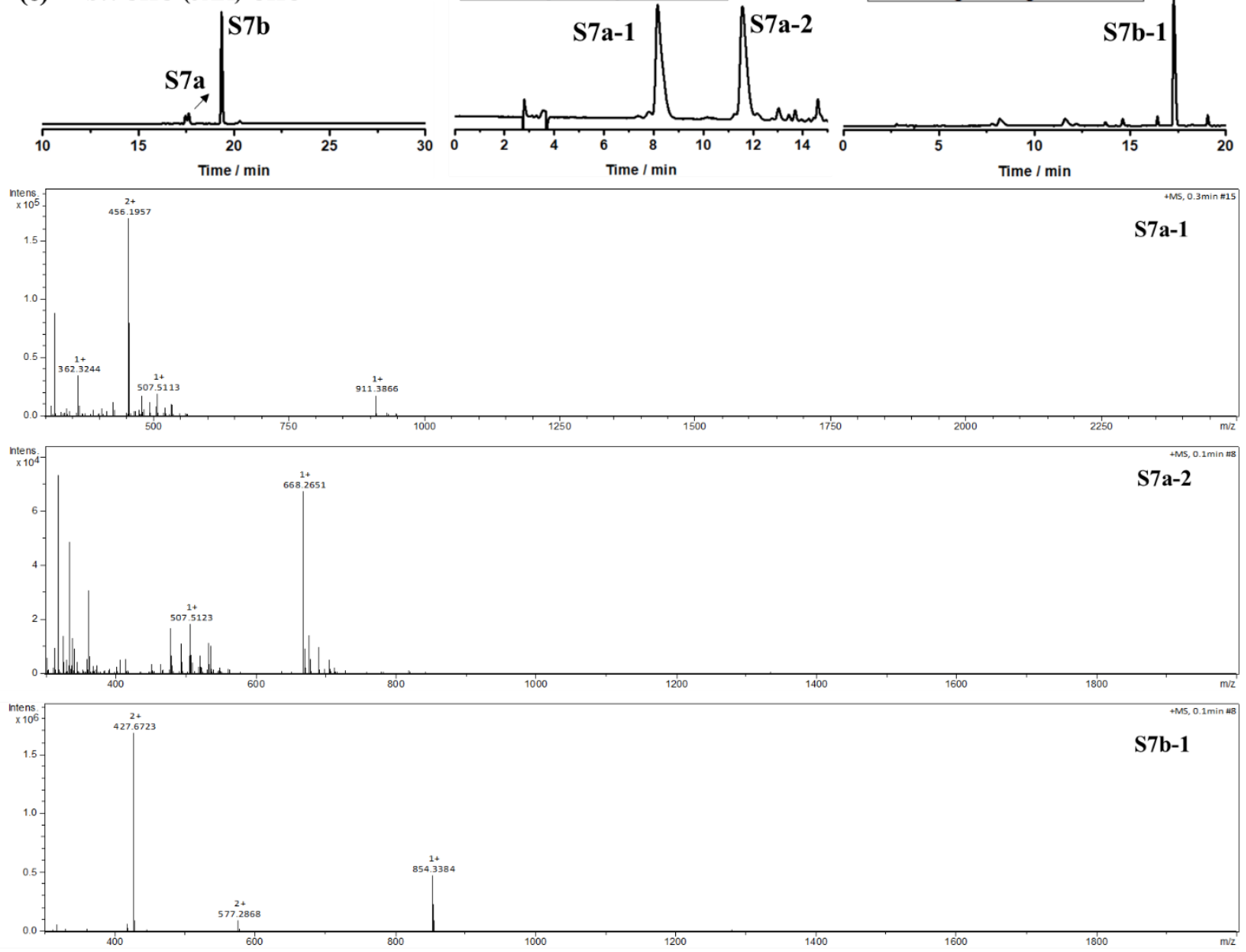

Fragment analysis:

\begin{tabular}{|c|c|c|c|c|}
\hline Peak No. & Sequence & Disulfide pairing & $\mathrm{m} / \mathrm{z}$ expected & $\mathrm{m} / \mathrm{z}$ found \\
\hline S7a-1 & $\begin{array}{r}\text { H-WGÇK } \\
\text { GGGCK }\end{array}$ & $1-3$ & $911.38^{1+}$ & $911.39^{1+}$ \\
\hline S7a-2 & $\begin{array}{l}\text { ÇGK } \\
\text { CGW-NH2 }\end{array}$ & $2-4$ & $668.26^{1+}$ & $668.26^{2+}$ \\
\hline S7-b-1 & $\underset{\text { H-WGC̣K }}{\text { CGW-NH2 }}$ & $1-4$ & $854.34^{1+}$ & $854.34^{1+}$ \\
\hline
\end{tabular}



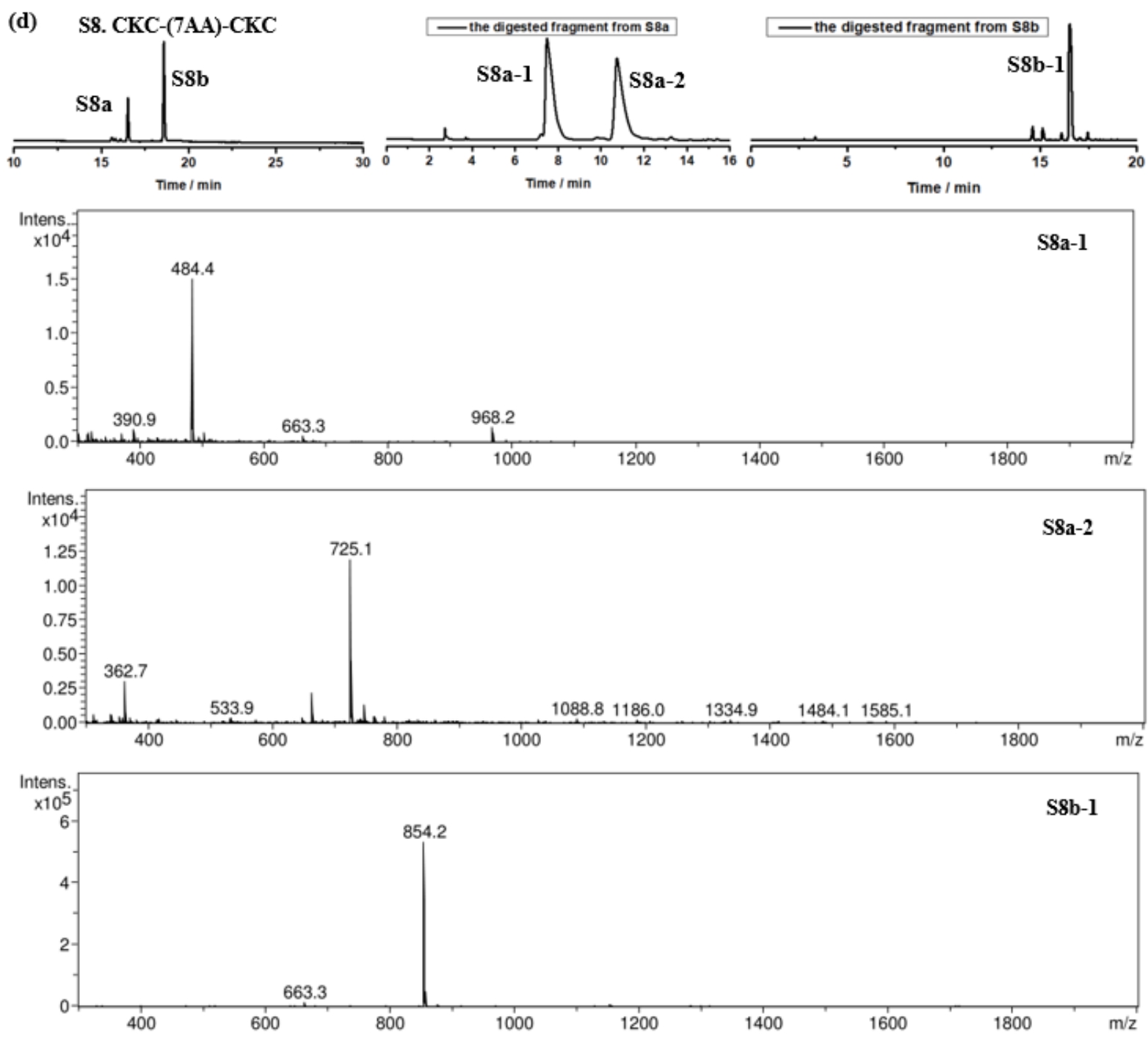

Fragment analysis:

\begin{tabular}{ccccc}
\hline Peak No. & Sequence & Disulfide pairing & $\mathrm{m} / \mathrm{z}$ expected & $\mathrm{m} / \mathrm{z}$ found \\
\hline S8a-1 & $\begin{array}{c}\text { H-WGCK } \\
\text { GGGGCK } \\
\text { S8a-2 }\end{array}$ & $1-3$ & $968.4^{1+}$ & $968.2^{1+}$ \\
CGGK \\
CGW-NH2 \\
S8-b-1
\end{tabular}



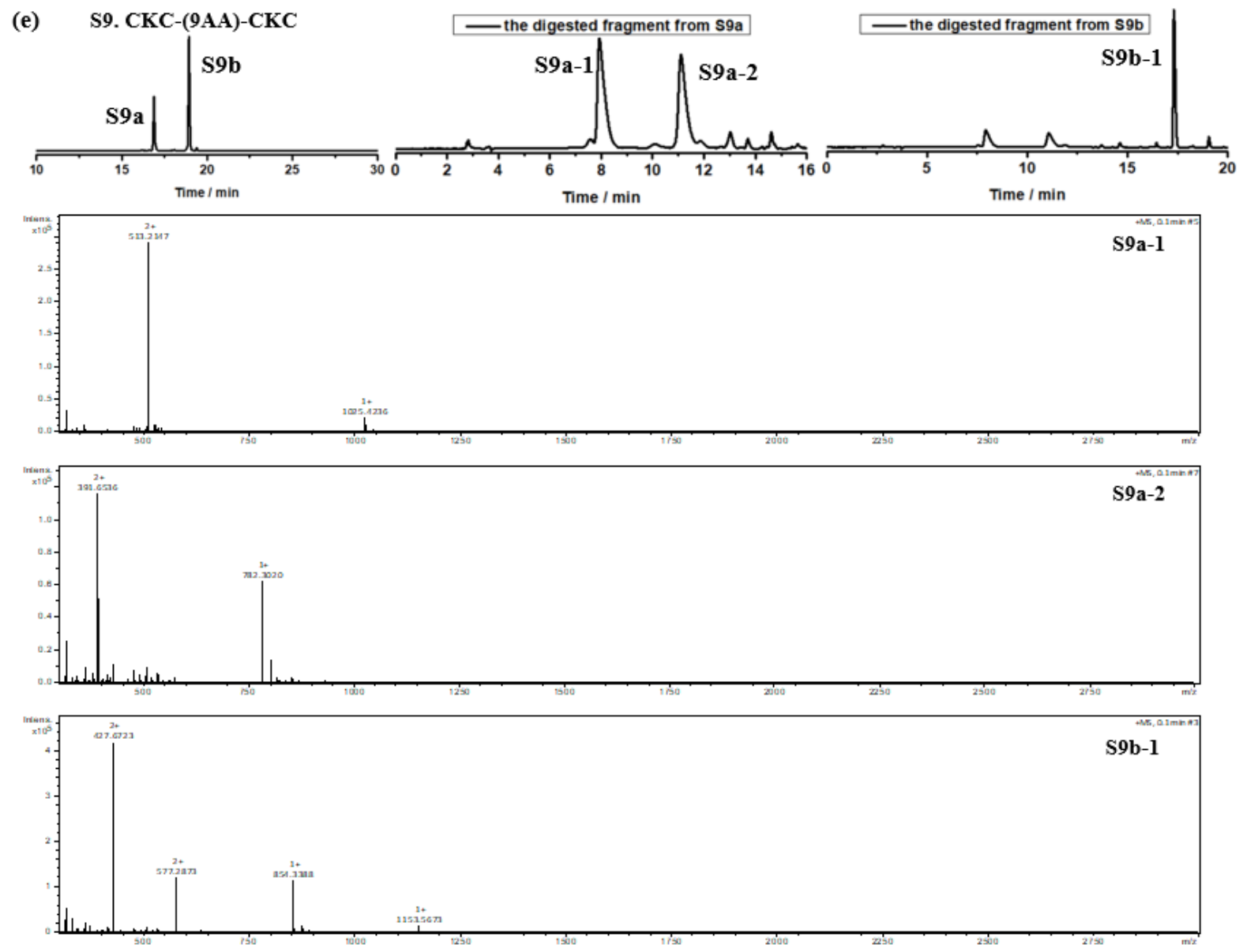

Fragment analysis:

\begin{tabular}{|c|c|c|c|c|}
\hline Peak No. & Sequence & Disulfide pairing & m/z expected & $\mathbf{m} / \mathbf{z}$ found \\
\hline S9a-1 & $\begin{array}{r}\text { H-WGCYK } \\
\text { GGGGGCK }\end{array}$ & $1-3$ & $1025.42^{1+}$ & $1025.42^{1+}$ \\
\hline S9a-2 & $\begin{array}{l}\text { CGGGK } \\
\text { CGW-NH2 }\end{array}$ & $2-4$ & $782.30^{1+}$ & $782.30^{1+}$ \\
\hline S9b-1 & $\begin{array}{l}\text { H-WGCKK } \\
\qquad \text { CGW-NH2 }\end{array}$ & $1-4$ & $854.34^{1+}$ & $854.34^{1+}$ \\
\hline
\end{tabular}


(f) S10. CKC-(13AA)-CKC
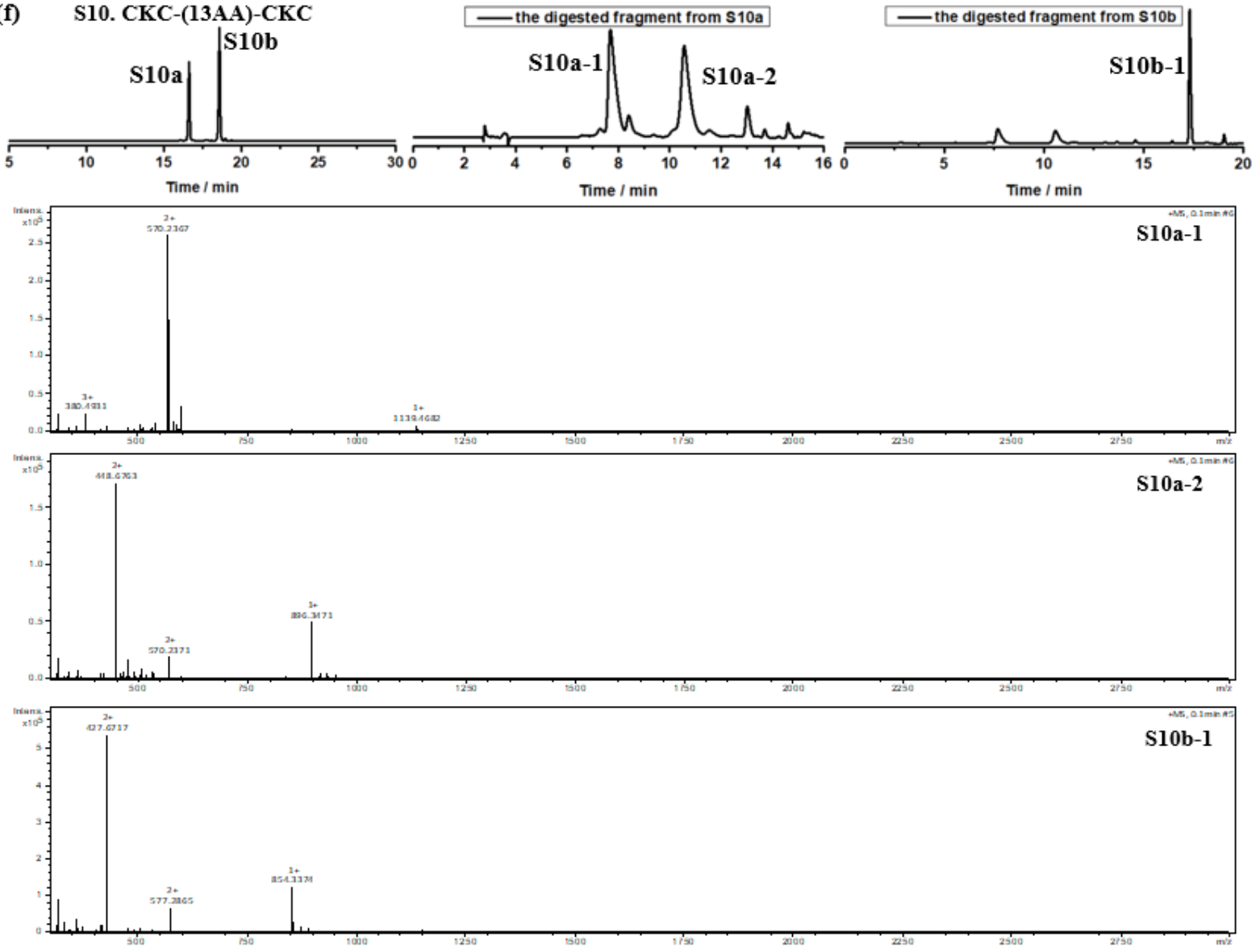

Fragment analysis:

\begin{tabular}{|c|c|c|c|c|}
\hline Peak No. & Sequence & Disulfide pairing & m/z expected & $\mathrm{m} / \mathrm{z}$ found \\
\hline S10a-1 & $\begin{array}{r}\text { H-WGCWK } \\
\text { GCK }\end{array}$ & $1-3$ & $1139.46^{1+}$ & $1139.47^{1+}$ \\
\hline S10a-2 & $\begin{array}{l}\text { CGGGGGK } \\
\text { ' } \mathrm{CWW}-\mathrm{NH}_{2}\end{array}$ & $2-4$ & $896.34^{1+}$ & $896.35^{1+}$ \\
\hline S10b-1 & $\begin{array}{l}\text { H-WGCCK } \\
\qquad \text { CGW-NH2 }\end{array}$ & $1-4$ & $854.34^{1+}$ & $854.34^{1+}$ \\
\hline
\end{tabular}



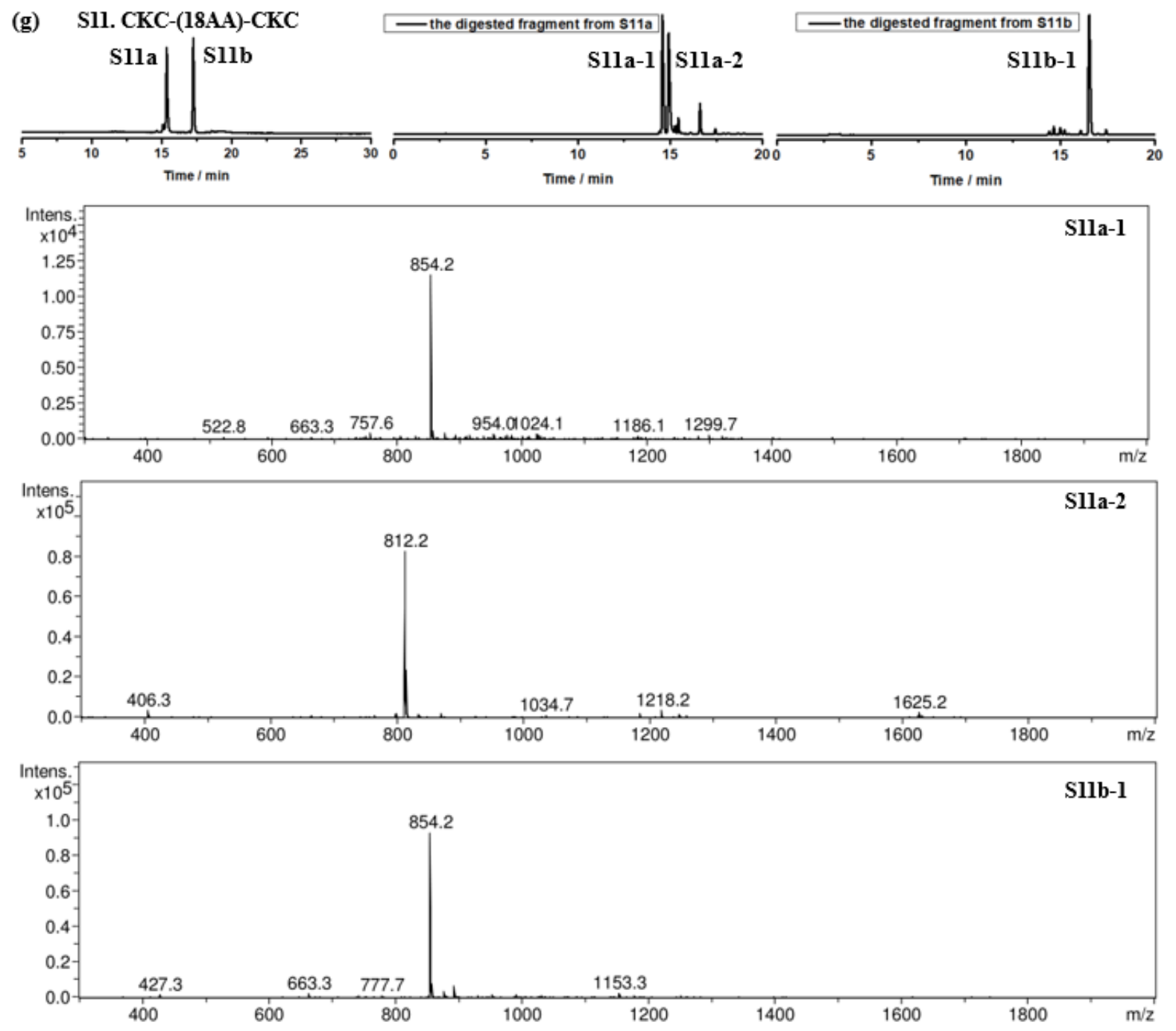

Fragment analysis:

\begin{tabular}{ccccc}
\hline Peak No. & Sequence & Disulfide pairing & $\mathrm{m} / \mathrm{z}$ expected & $\mathrm{m} / \mathrm{z}$ found \\
\hline S11a-1 & $\begin{array}{c}\text { H-WGCK } \\
\text { GGCK }\end{array}$ & $1-3$ & $854.4^{1+}$ & $\mathbf{8 5 4 . 2}^{1+}$ \\
S11a-2 & $\begin{array}{c}\text { CSGGK } \\
\text { CGW-NH2 }\end{array}$ & $2-4$ & $812.3^{1+}$ & $812.2^{1+}$ \\
S11b-1 & $\begin{array}{c}\text { H-WGCK } \\
\text { CGW-NH2 }\end{array}$ & $1-4$ & $854.3^{1+}$ & $854.2^{1+}$ \\
\hline
\end{tabular}

Fig S4. (a) The sequence information of peptides S7-11. (b) Chromatograms showing the oxidation of S7, S8, S9, S10 and S11 in phosphate buffer (100 mM, pH 7.4) containing $0.5 \mathrm{mM}$ oxidized glutathione (GSSG). (c) Confirmation on disulfide pairing of S7 by the mass spectra of digested fragments. (d) Confirmation on disulfide pairing of $\mathbf{S 8}$ by the mass spectra of digested fragments. (e) Confirmation on disulfide pairing of $\mathbf{S 9}$ by the mass spectra of digested fragments. (f) Confirmation 
on disulfide pairing of $\mathbf{S 1 0}$ by the mass spectra of digested fragments. (g) Confirmation on disulfide pairing of $\mathbf{S 1 1}$ by the mass spectra of digested fragments. 
2.5 Analysis of disulfide pairing of 7

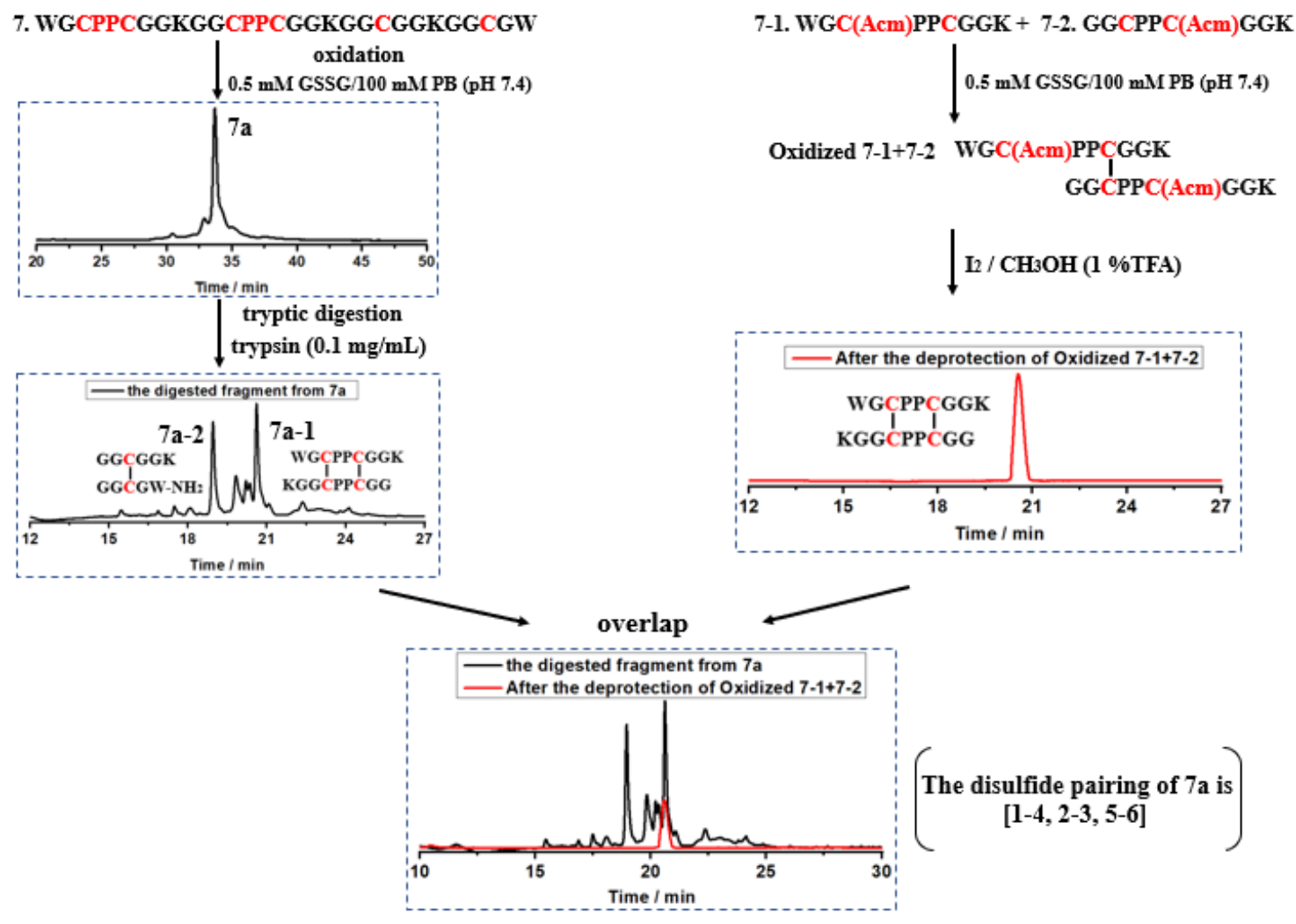



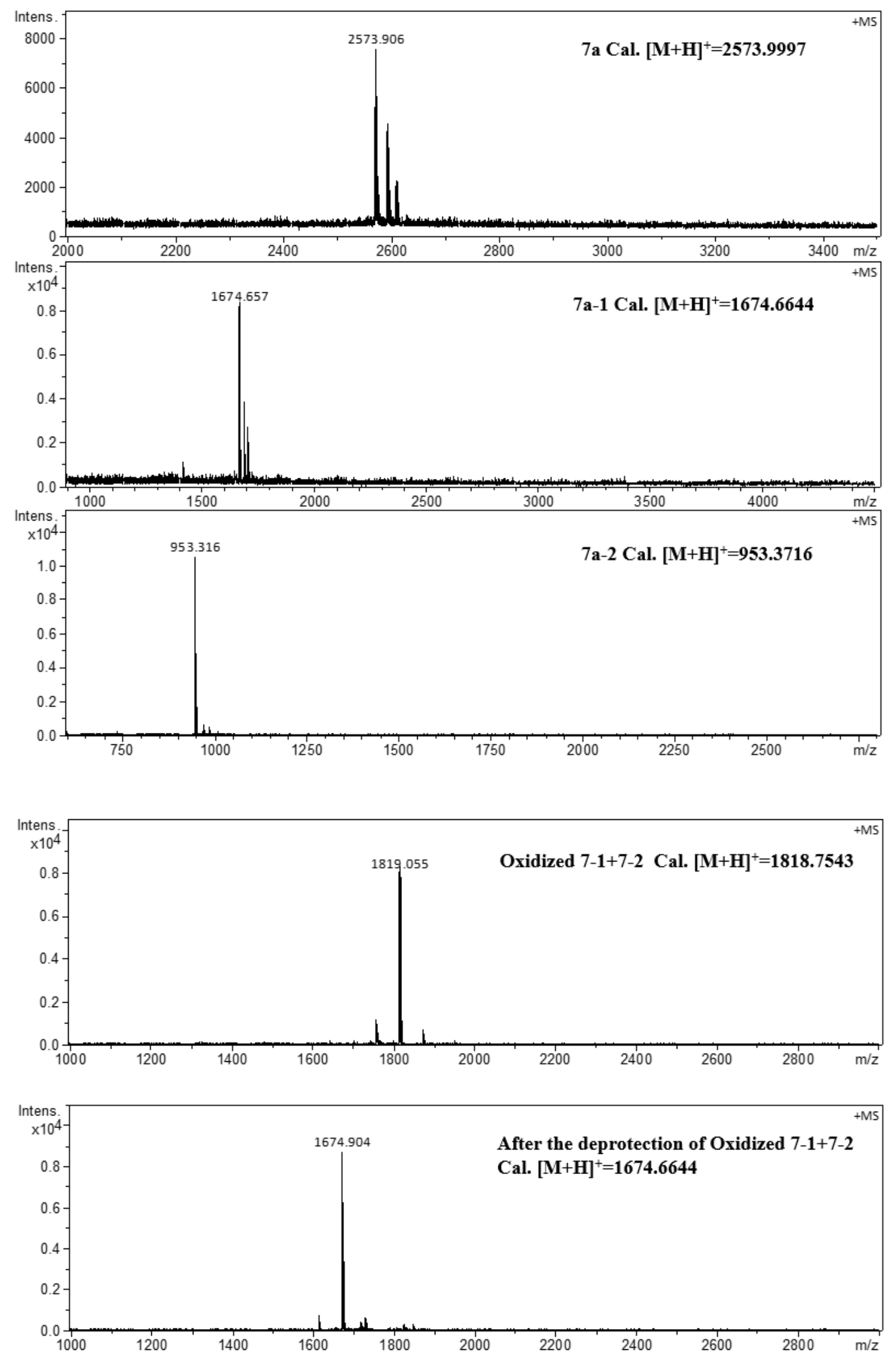

Fig S5. Confirmation on disulfide pairing of 7 by tryptic digestion and analysis of the peptide fragments by HPLC and mass spectrometry. 


\subsection{Analysis of disulfide pairing of 8}

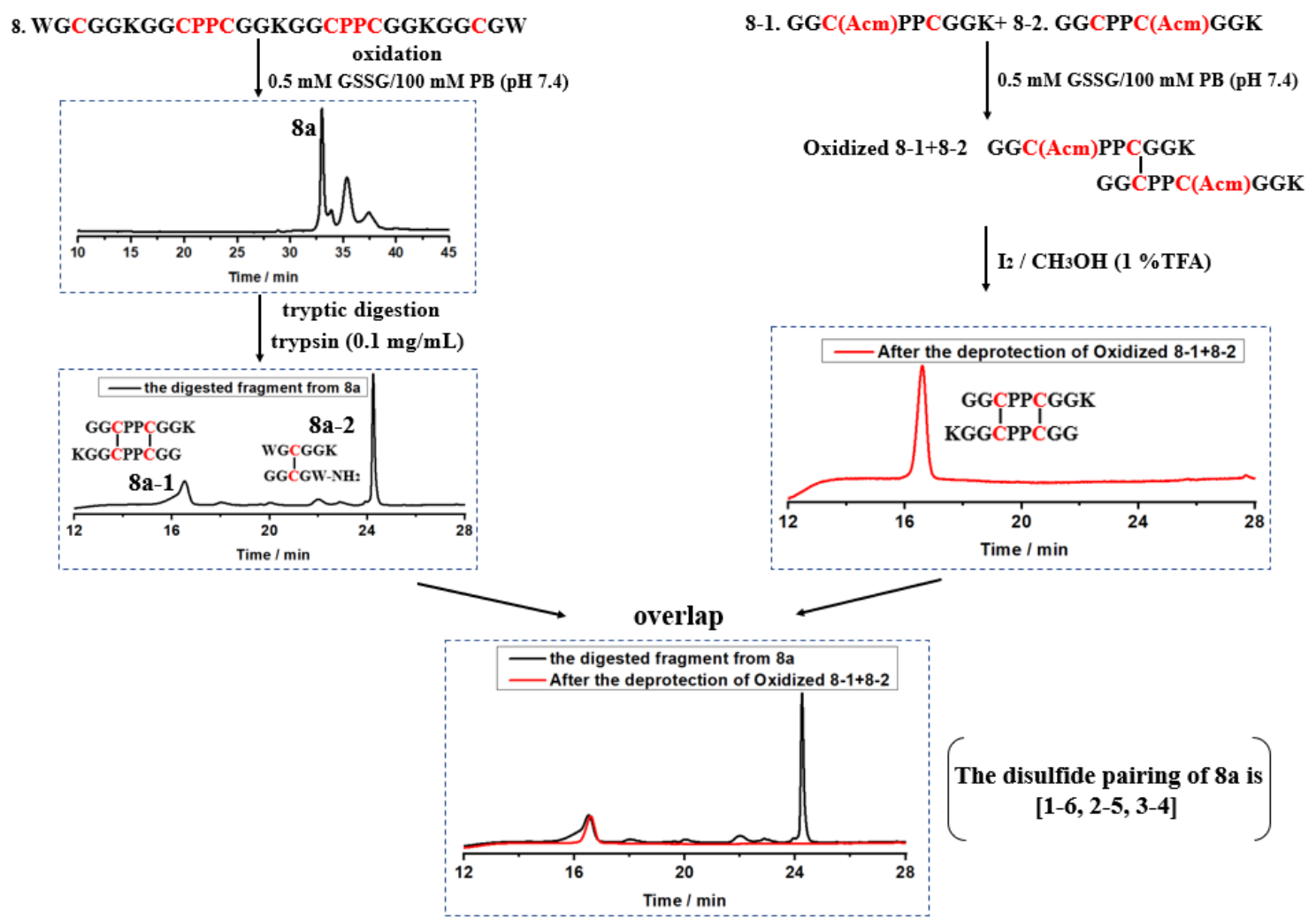



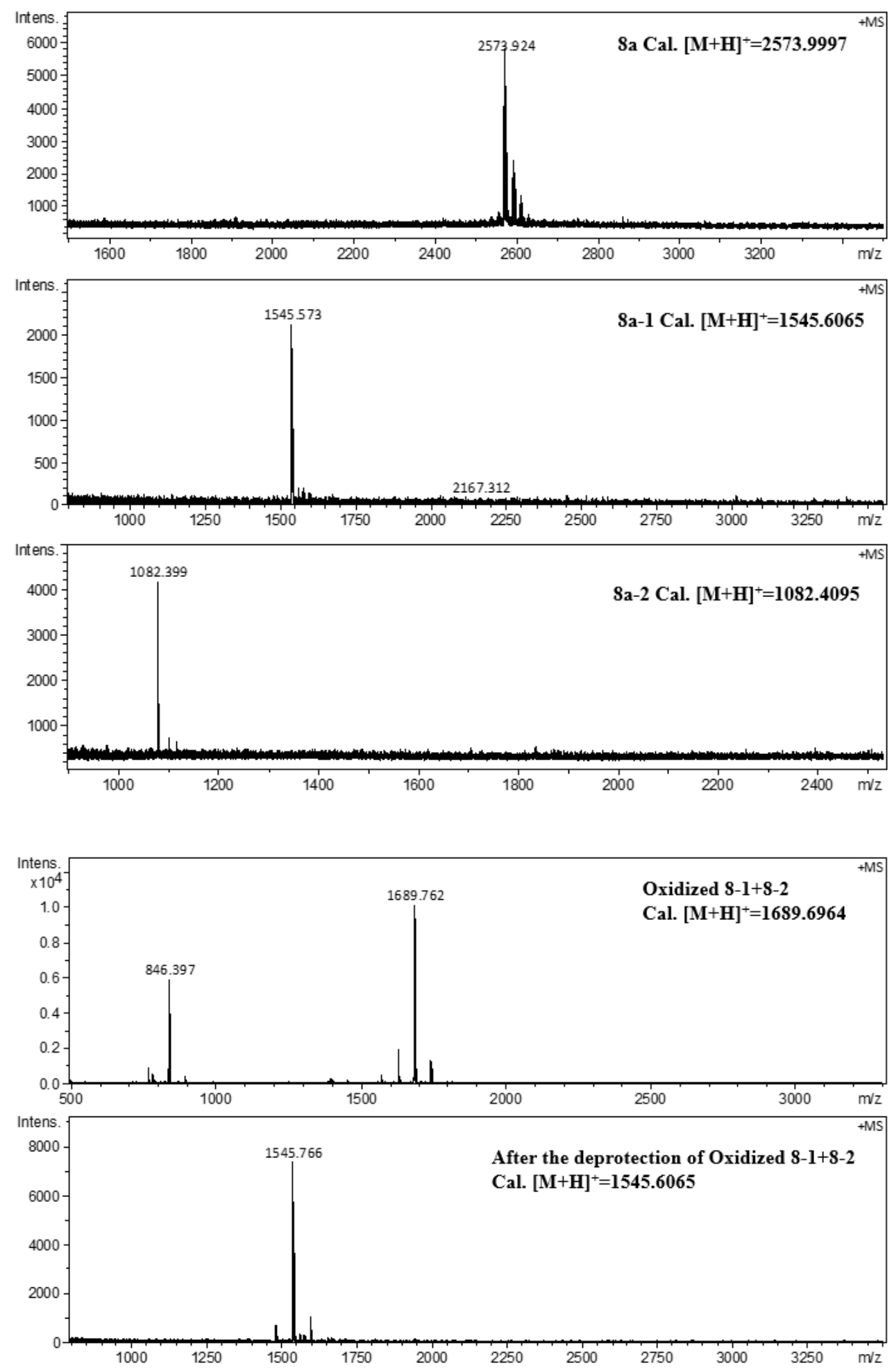

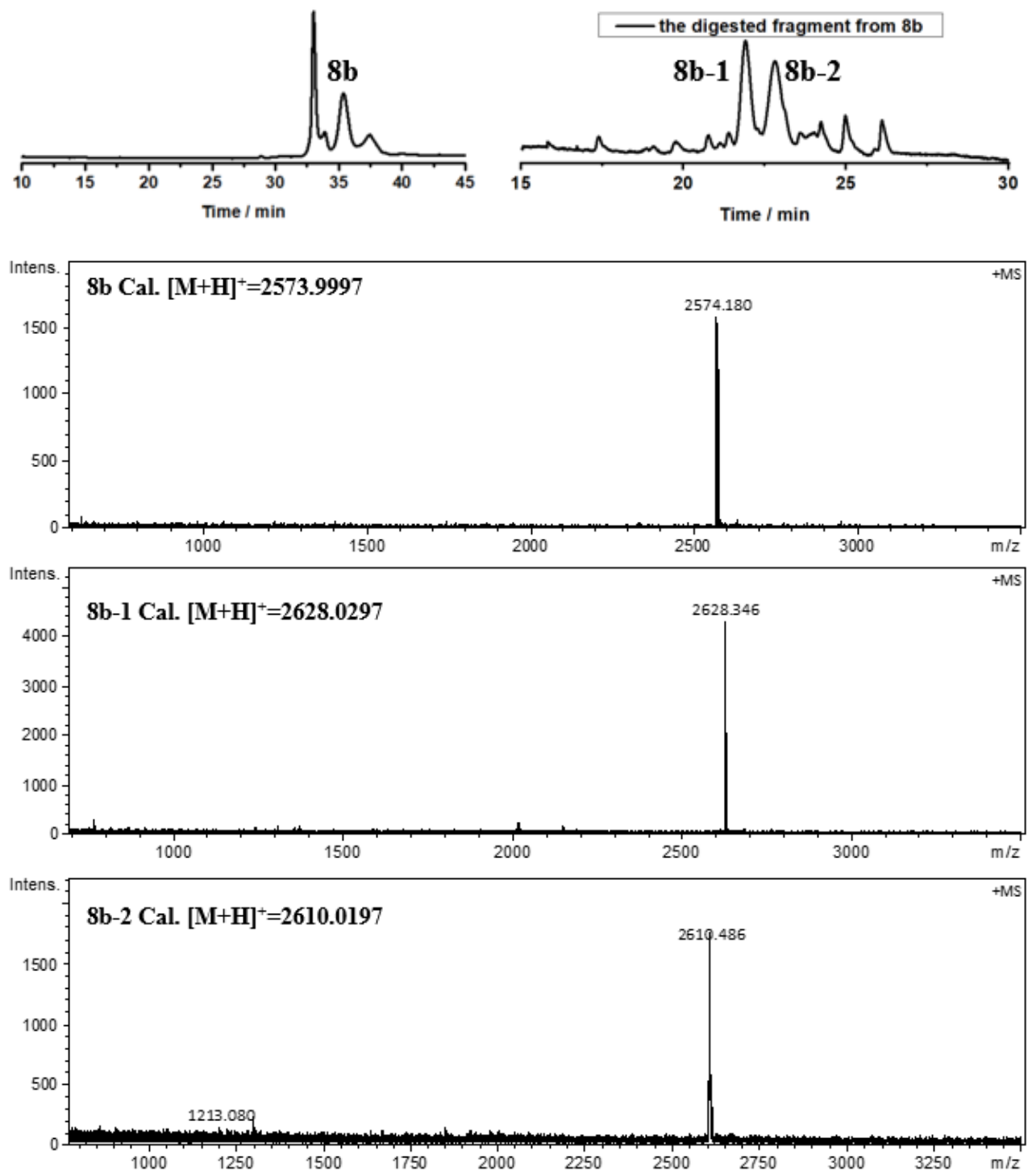

Fig S6. Confirmation on disulfide pairing of $\mathbf{8}$ by tryptic digestion and analysis of the peptide fragments by HPLC and mass spectrometry. The digested fragments of $\mathbf{8 b}$ indicate the formation of mixed disulfides between the CPPC motifs and the isolated cysteine residues. 


\subsection{Analysis of disulfide pairing of 9}

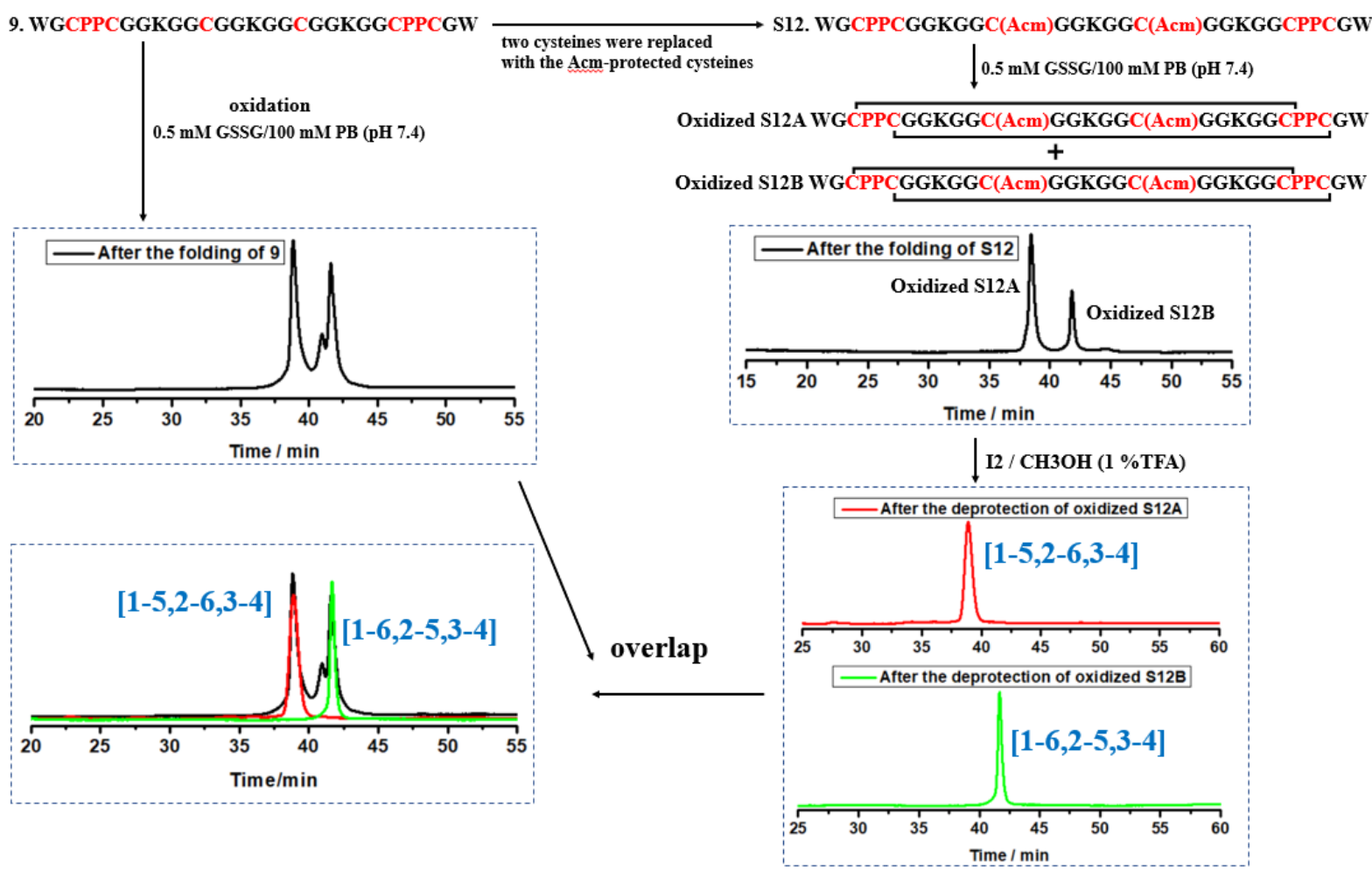

9. WGCPPCGGKGGCGGKGGCGGKGGCPPCGW oxidation $0.5 \mathrm{mM}$ GSSG/100 mM PB (pH 7.4)

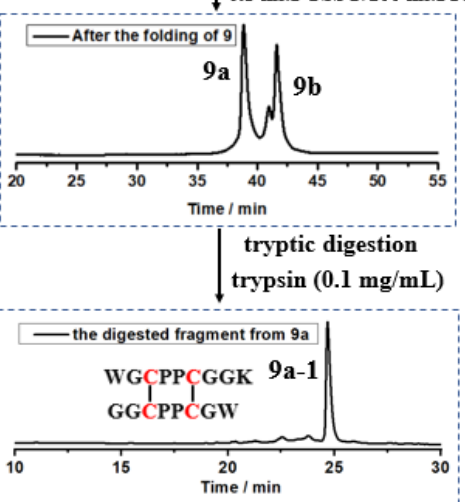

9-1. WGC(Acm)PPCGGK + 9-2. GGC(Acm)PPCGW $0.5 \mathrm{mM}$ GSSG/100 mM PB (pH 7.4)

Oxidized 9-1+9-2 WGC(Acm)PPCGGK GGC(Acm)PPCGW I2 / CH3 OH (1 \%TFA)

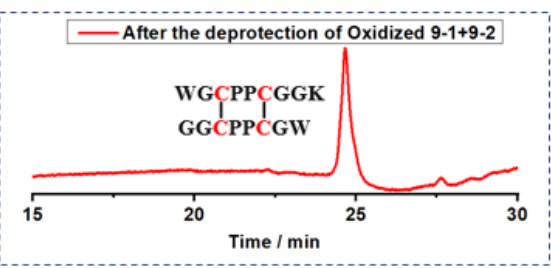

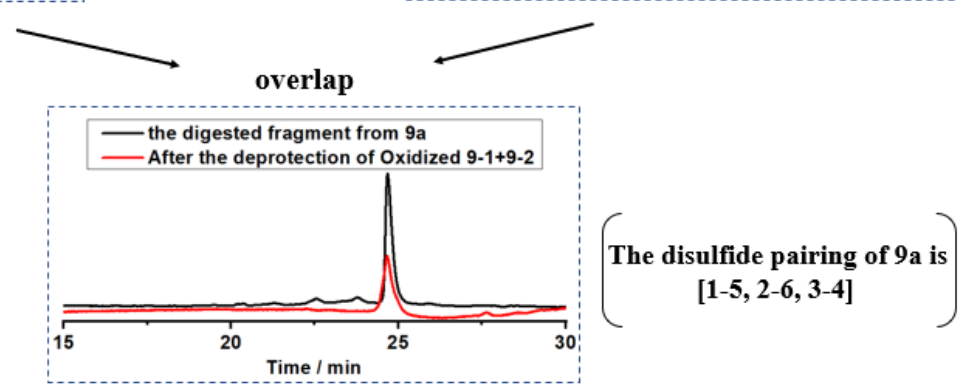



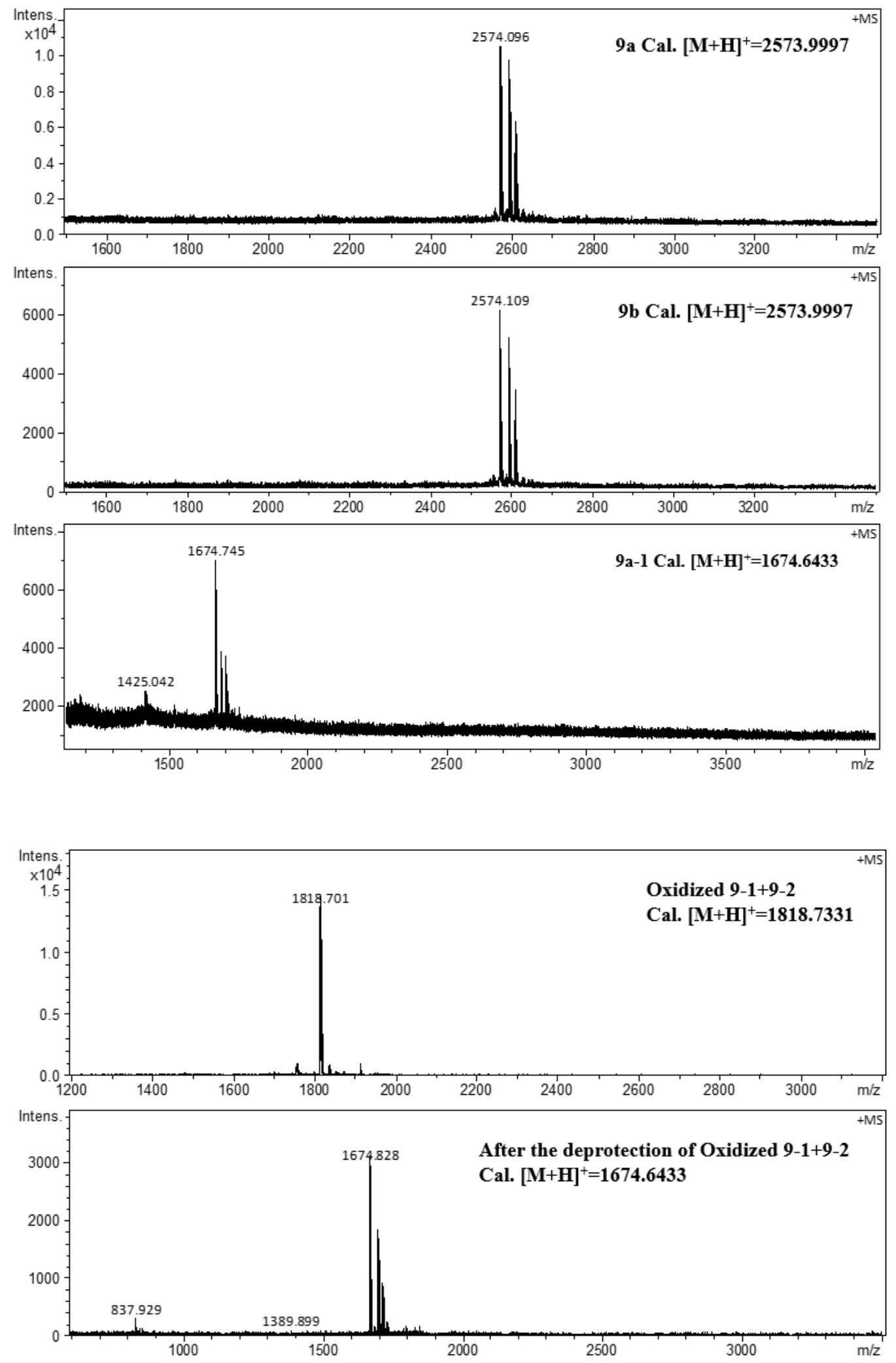

Fig S7. Confirmation on disulfide pairing of 9 through orthogonal protecting group strategy and tryptic digestion LC-MS analysis. 


\subsection{Analysis of disulfide pairing of $\mathbf{1 0}$}

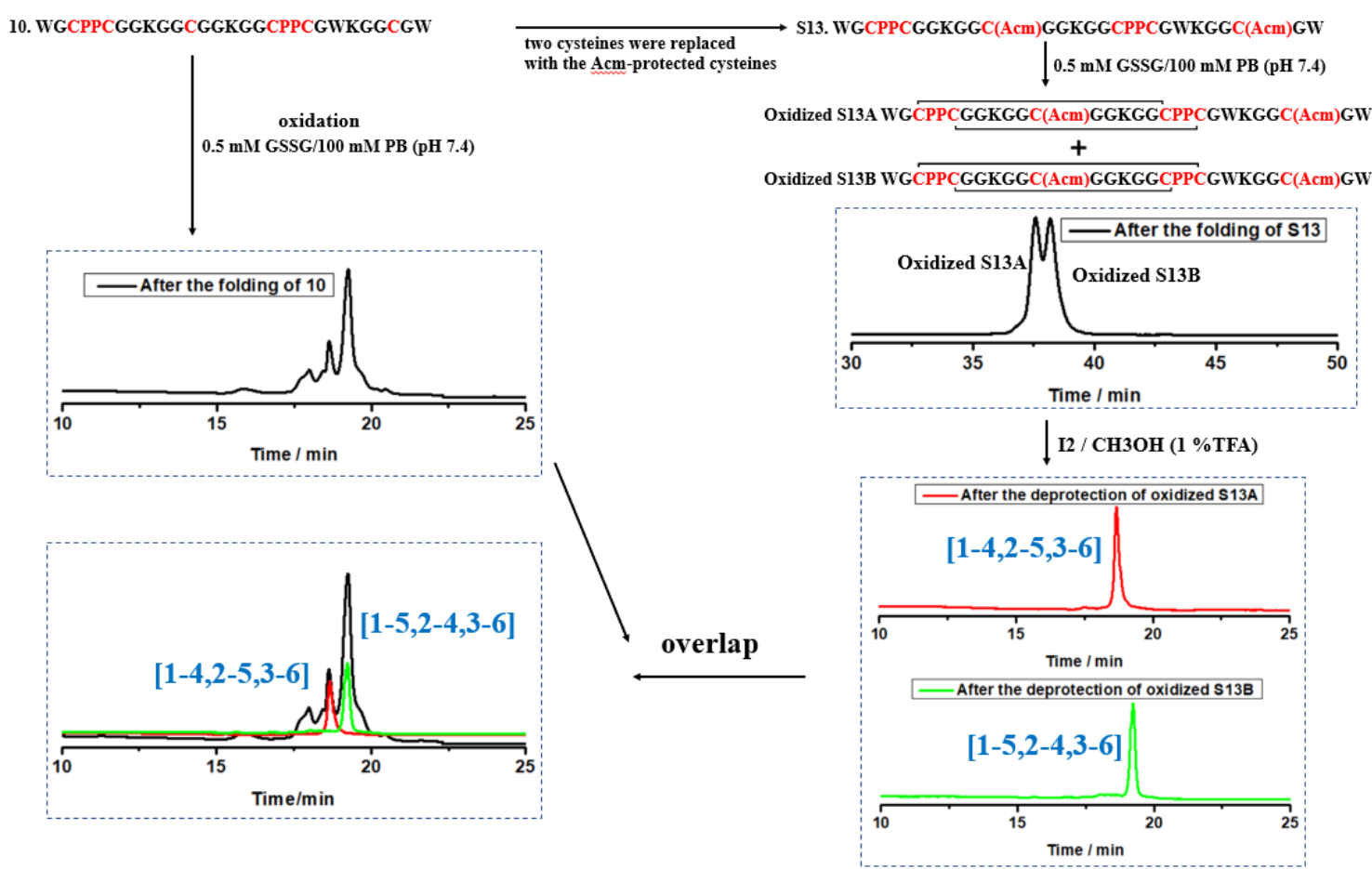

10. WGCPPCGGKGGCGGKGGCPPCGWKGGCGW oxidation $0.5 \mathrm{mM}$ GSSG/100 mM PB (pH 7.4)

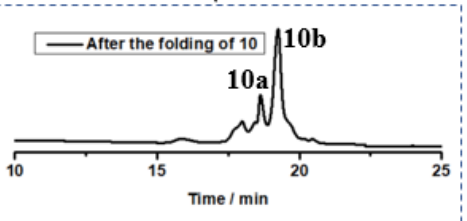

tryptic digestion

trypsin $(0.1 \mathrm{mg} / \mathrm{mL})$

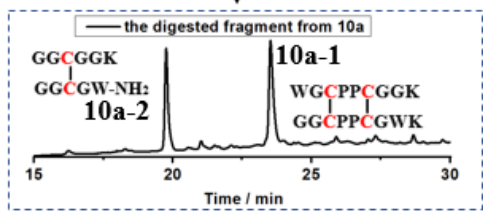

10-1. WGC(Acm)PPCGGK + 10-2. GGC(Acm)PPCGWK $0.5 \mathrm{mM}$ GSSG/100 mM PB (pH 7.4)

Oxidized 10-1+10-2 WGC(Acm)PPCGGK GGC(Acm)PPCGWK

$\mathrm{I}_{2} / \mathrm{CH}_{3} \mathrm{OH}(1 \% \mathrm{TFA})$

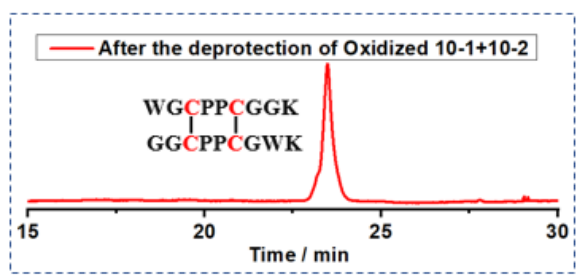

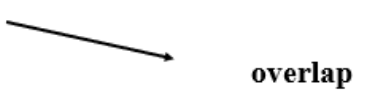

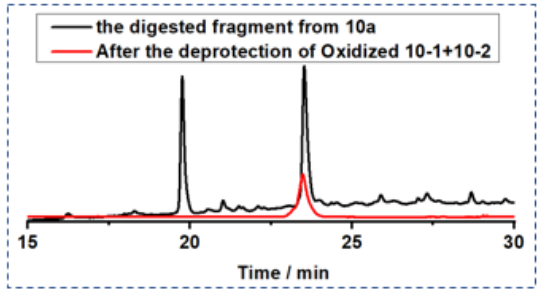

The disulfide pairing of $10 \mathrm{a}$ is $[1-4,2-5,3-6]$ 

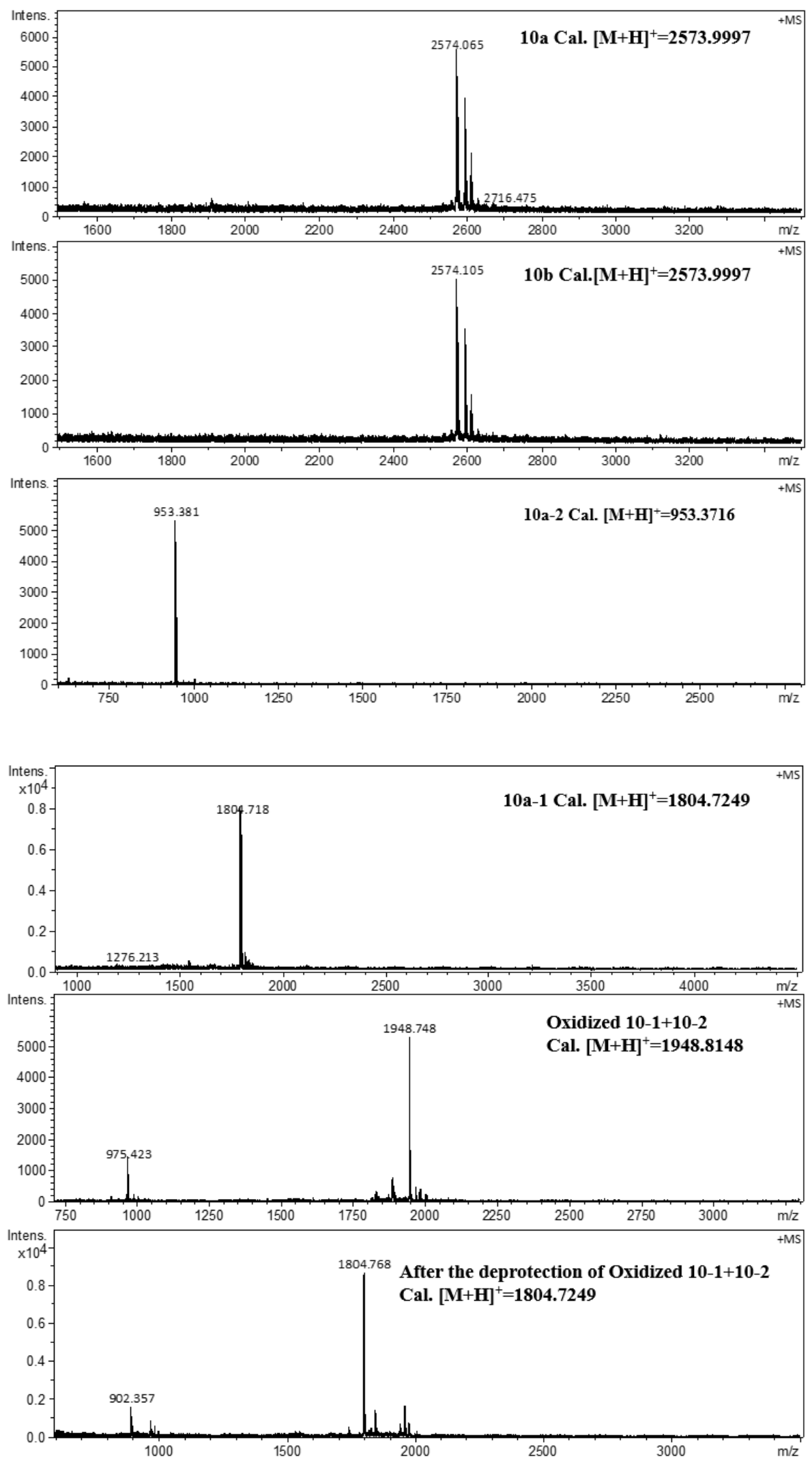

Fig S8. Confirmation on disulfide pairing of $\mathbf{1 0}$ through orthogonal protecting group strategy and tryptic digestion LC-MS analysis. 


\subsection{Analysis of disulfide pairing of 11}

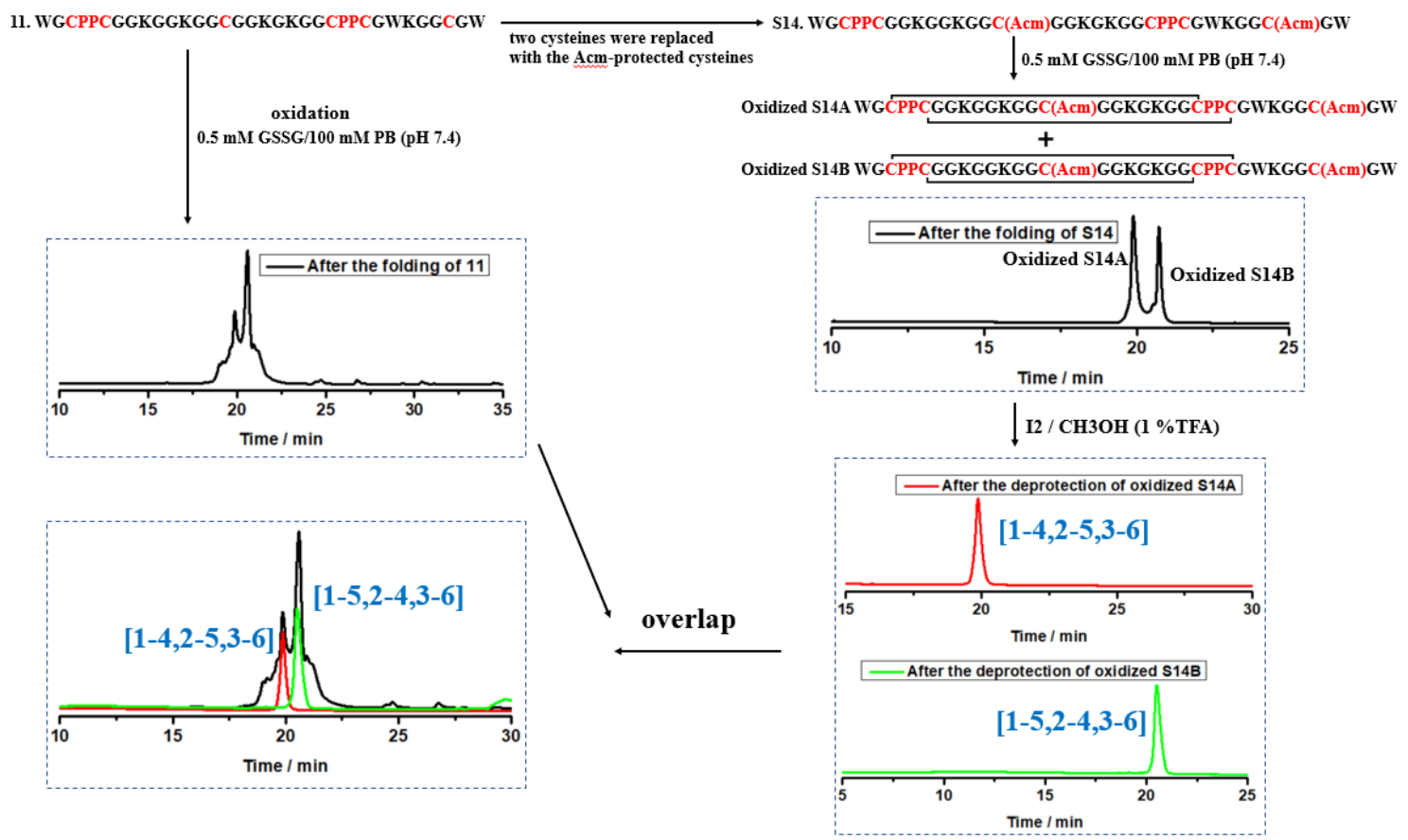

11. WGCPPCGGKGGKGGCGGKGKGGCPPCGWKGGCGW

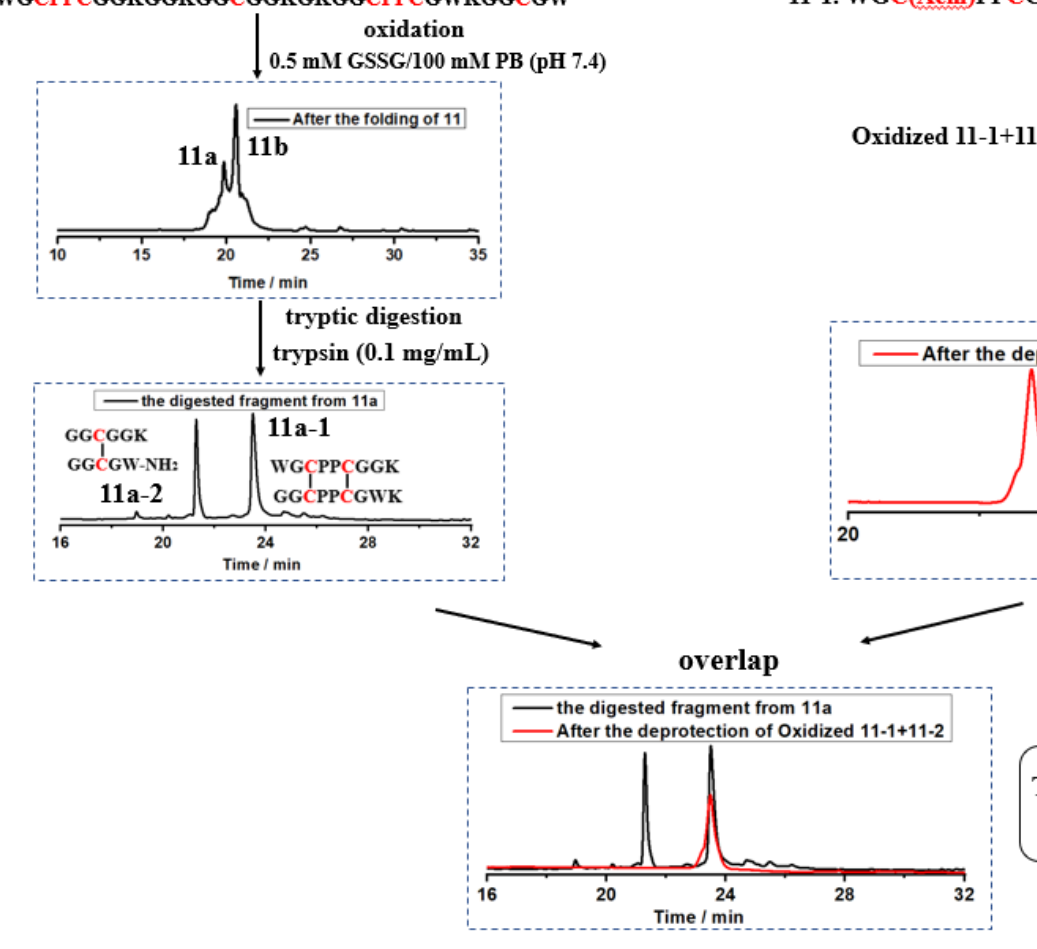

11-2. GGC(Acm)PPCGWK $0.5 \mathrm{mM}$ GSSG/100 mM PB (pH 7.4)

WGC(Acm)PPCGGK GGC(Acm)PPCGWK

I 2 CH3OH (1 \%TFA)

The disulfide pairing of 11a is $[1-4,2-5,3-6]$ 

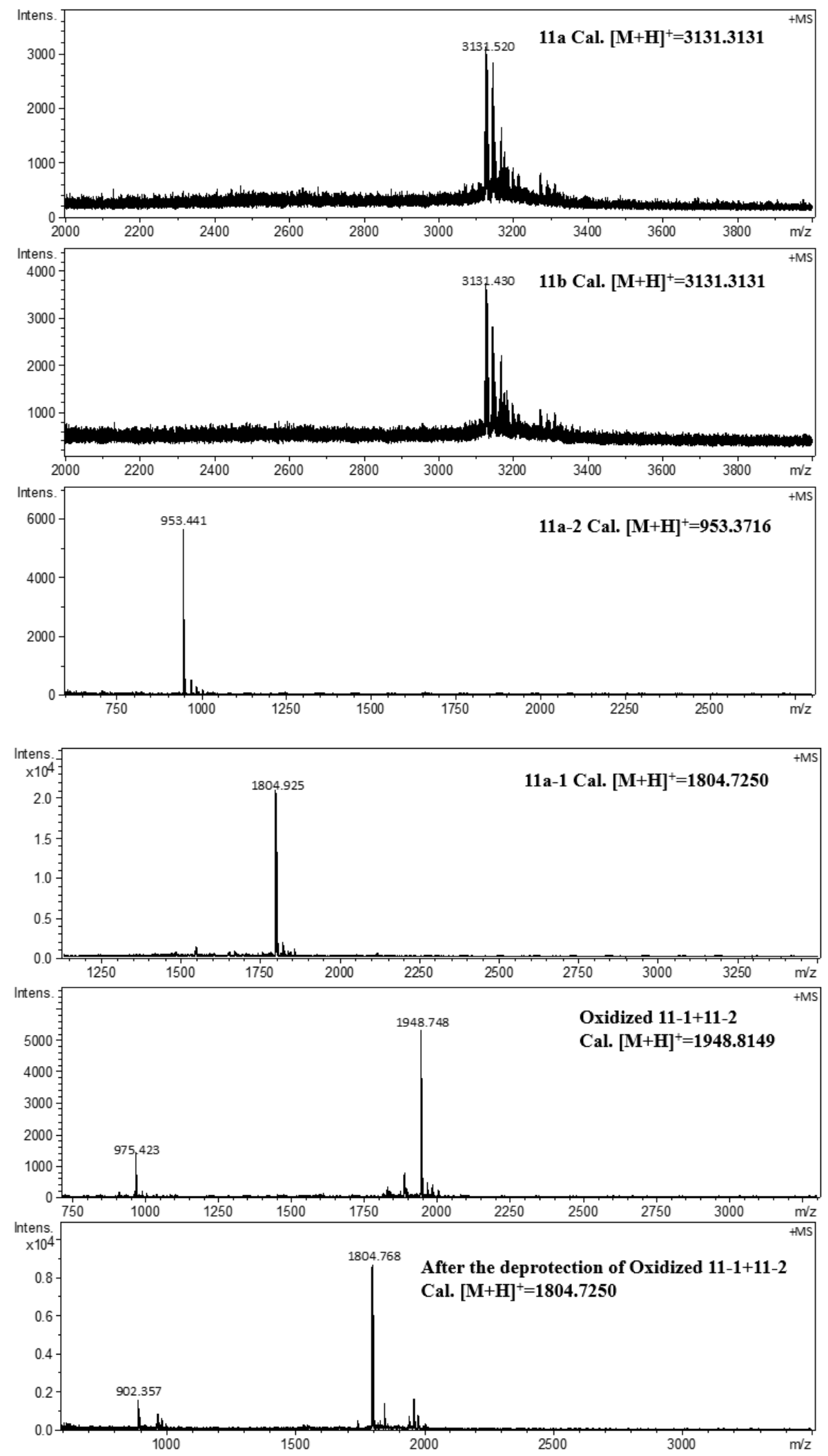

Fig S9. Confirmation on disulfide pairing of $\mathbf{1 1}$ through orthogonal protecting group strategy and tryptic digestion LC-MS analysis. 


\subsection{Alignment of 60 clones' sequences from the library}

\begin{tabular}{|c|c|}
\hline s: & Sequence: \\
\hline 1 & C P P CVEWY L C P GQP P CMH S L E C P F \\
\hline 2 & CP PCAS L S LCALLSKCP P SAVCPPC \\
\hline 3 & CP PCPKDHF CLRPKLCR I L F T P PC \\
\hline 4 & CP PCDHSKVCFANSACSNSTRCP PC \\
\hline 5 & C P P CGVVAVCR T RHHCMKE SWC P PC \\
\hline 6 & CP PCGVVAVCRHEA S CMKE SWCP PC \\
\hline 7 & C P P CLRAARCS R T P R CQWT QVCP PC \\
\hline 8 & CP PCLHNTWCHES SECLAF \\
\hline 9 & CP PCLRRACCS \\
\hline 10 & CPPCI E L P VCLN \\
\hline 11 & CPPCP S GLRCL T \\
\hline 12 & CP PCRQE LECSES F VCLLLAT \\
\hline 13 & CP PCPWVF DCT T DE E CRGS Y \\
\hline 14 & CP PCAR P D T C \\
\hline 15 & CPPCIVFQSCLI THFCIY \\
\hline 16 & CPPCREYLDCT TSALCP PSI WCPPC \\
\hline 17 & CP PCE P RR S C P KG P HCL VVNT C P PC \\
\hline 18 & CP PCRWER I CHAQL K CQL D P E C P PC \\
\hline 19 & CP PCPACLECPVP LRCS LQNQCP PC \\
\hline 20 & CP PCR F C GGCGYGDRCP P HS P C P PC \\
\hline 21 & CP P CE P GG S C YRRAWC F HQE E C P PC \\
\hline 22 & CP PCPRKS S CYP ERRCEVTDACP PC \\
\hline 23 & C P P CV P EWQCDD P AL C I Y I YMC P PC \\
\hline 24 & CP PCWF RE GCCNE T F C F I MR I CP PC \\
\hline 25 & C P PCG S QR L C L DAWE CV P P L L C P PC \\
\hline 26 & CP P CQT SWR CL L I S YCP DR T KCP PC \\
\hline 27 & CP PCATLGRCLSRAGCRLYP TCP PC \\
\hline 28 & CPPCGCYETCFCPEFCS F LWNCPPC \\
\hline 29 & \\
\hline O & F TVSNCVLGESCP \\
\hline
\end{tabular}

\begin{tabular}{|c|c|}
\hline s: & ence: \\
\hline 31 & CP PCCLRRVCPSDVLCEHSRMCP \\
\hline 32 & CP PCR I EWT CK S CQHCYE YMT CP PC \\
\hline 33 & CP PCR SCAPCVSRGPCASAL I CPI \\
\hline 34 & CP PCE PCGTCPGAEGCEARE ECPPC \\
\hline 35 & CP PCL L GA T C L I MHP CGEAE L CP PC \\
\hline 36 & CP P C E L VMR CQY S Q P CCY P \\
\hline 37 & CPPCGANRGCRN \\
\hline 38 & CP PCPYAGLCLT \\
\hline 39 & CP PCS RA \\
\hline 40 & CP PCG \\
\hline 41 & CP PCT F \\
\hline 42 & CPPCR \\
\hline 43 & LQCHMG \\
\hline 44 & CP PC \\
\hline 45 & CPPCLG \\
\hline 46 & CPPCQG \\
\hline 47 & CP PCL E C F MC LMH \\
\hline 48 & CP PCESACLCRTNS YCE PAE PCP PC \\
\hline 49 & CPPCGAVPSCATAVVCS I F QNCPPC \\
\hline 50 & CL P F RECP PC \\
\hline 51 & E S I Q S CPPC \\
\hline 52 & C T F L T CP PC \\
\hline 53 & CP PCRH \\
\hline 5 & C L L F P R CA E E LWC P PC \\
\hline 5 & CP PCP CVR I CP S Y T \\
\hline 5 & CPPCF S L P QCF GL \\
\hline 5 & CP P C F R QDHC E AYY I CHMAV T C P PC \\
\hline 5 & CPPCLRLVICIFFYCCFYYAFCPPC \\
\hline $\mathbf{3}$ & \\
\hline 60 & GCACEYTRVC \\
\hline
\end{tabular}

Fig S10. The 60 clones' sequences from the library. 
2.11 Alignment of the selected sequences

\begin{tabular}{|c|c|}
\hline Clones: & Sequence: \\
\hline M-1 & 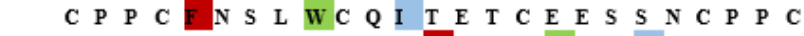 \\
\hline M-3 & C P P C C H G R P T C D S \\
\hline M-4 & C P P C H G R P T C D S \\
\hline M-5 & C P P C H G R P T C D S \\
\hline M-6 & C P P CSNSGGCETEWWCALDGTCP P C \\
\hline M-7 & C P P C H G R P T C D S F T N C WEL L T C P P C \\
\hline M-s & C P P C H R P TCDSFT N C WEL L T C P P C \\
\hline M-9 & C P P C H G R P T C D S ET N C WELL T C P P C \\
\hline M-11 & C P P C S N S G G C E TEWW C A L D G T C P P C \\
\hline M-12 & 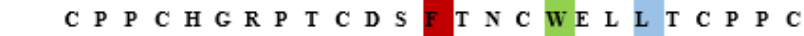 \\
\hline M-13 & C P P C H G R P T C D S F T N C WE L L T C P P C \\
\hline M-14 & C P P C H G R P T C D S F T N C WEL L T C P P C \\
\hline M-15 & C P P C H G R P T C D S E T N C WEL L T C P P C \\
\hline & Abundance \\
\hline & CPPCHGRPTCDSFTNCWELLT CPPC \\
\hline & CPPCSNSGGCFTIEWWCALDGTCPPC \\
\hline & CPPCFNSLWCQIITET CEE SSNCPPC \\
\hline
\end{tabular}

Fig S11. Sequence alignment of phage selection of SUMO-MDM2 (Conserved amino acid residues are highlighted in color). 


\subsection{Analysis of disulfide pairing of 12}

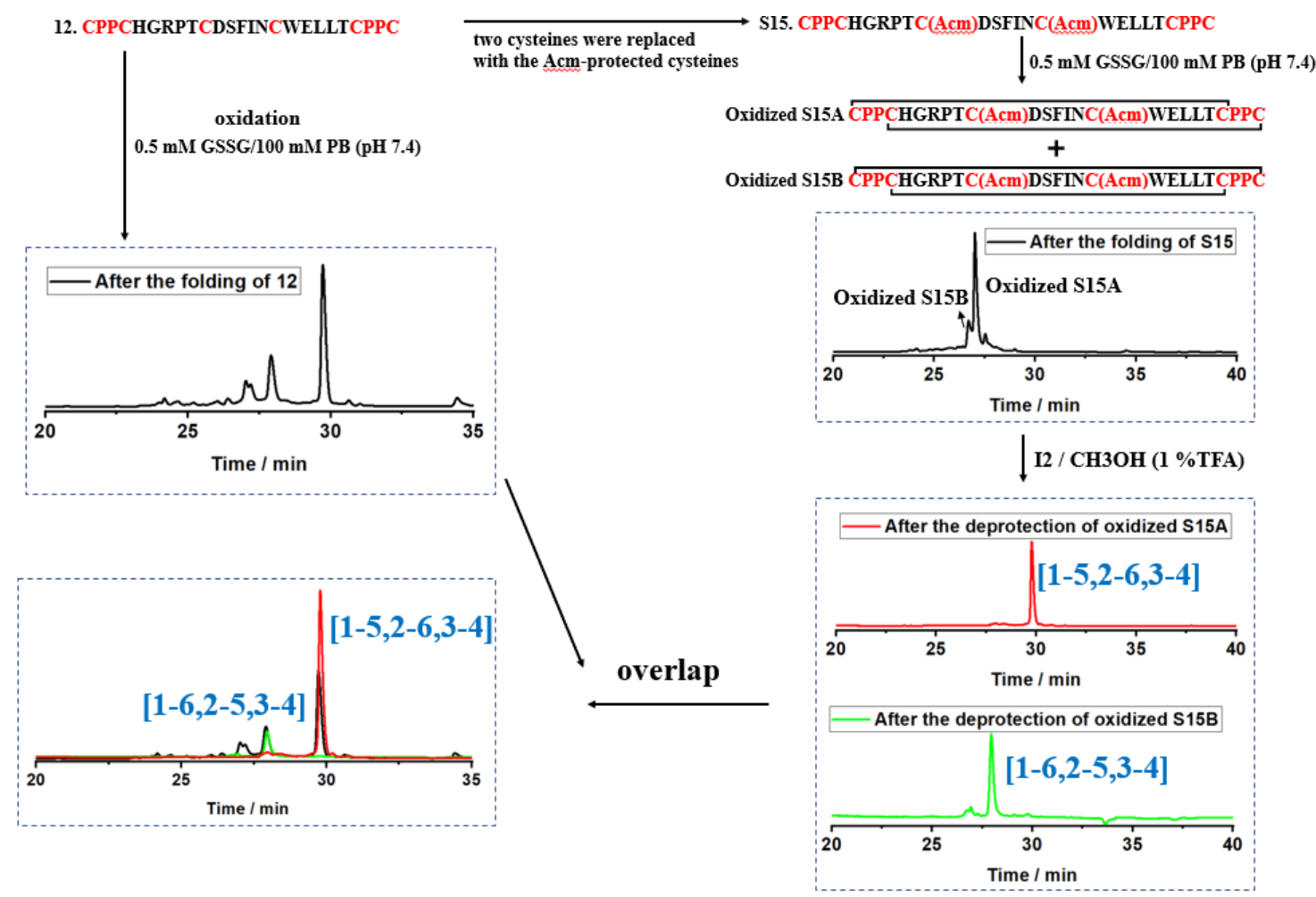

Fig S12. The flowchart to determine the disulfide pairing of $\mathbf{1 2}$. 


\subsection{Binding affinity of the oxidized peptide S16 to MDM2}

(a) S16. WGCPPCHGRPTCDSATNCAELLTCPPC

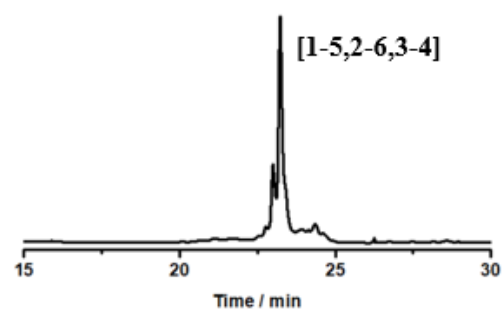

(b)

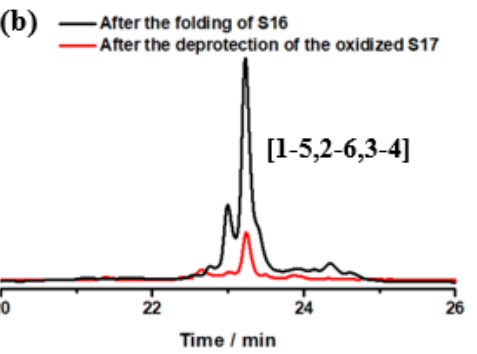

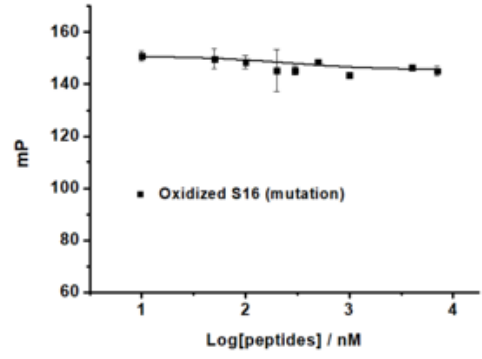

Fig S13. (a) Chromatogram showing the oxidation of $\mathbf{S 1 6}$ in phosphate buffer (100 mM, pH 7.4) containing $0.5 \mathrm{mM}$ oxidized glutathione (GSSG). (b) Chromatogram of products formed from the oxidative folding of S16 (black line) and chromatogram of the standard tricyclic peptide obtained from S17 (sequence: WGCPPCHGRPTC(Acm)DSATNC(Acm)AELLTCPPC) using the orthogonal protecting group strategy (red line). (c) FP competition curves showing the binding of oxidized S16 to MDM2. 


\subsection{Analysis of disulfide pairing of 13}

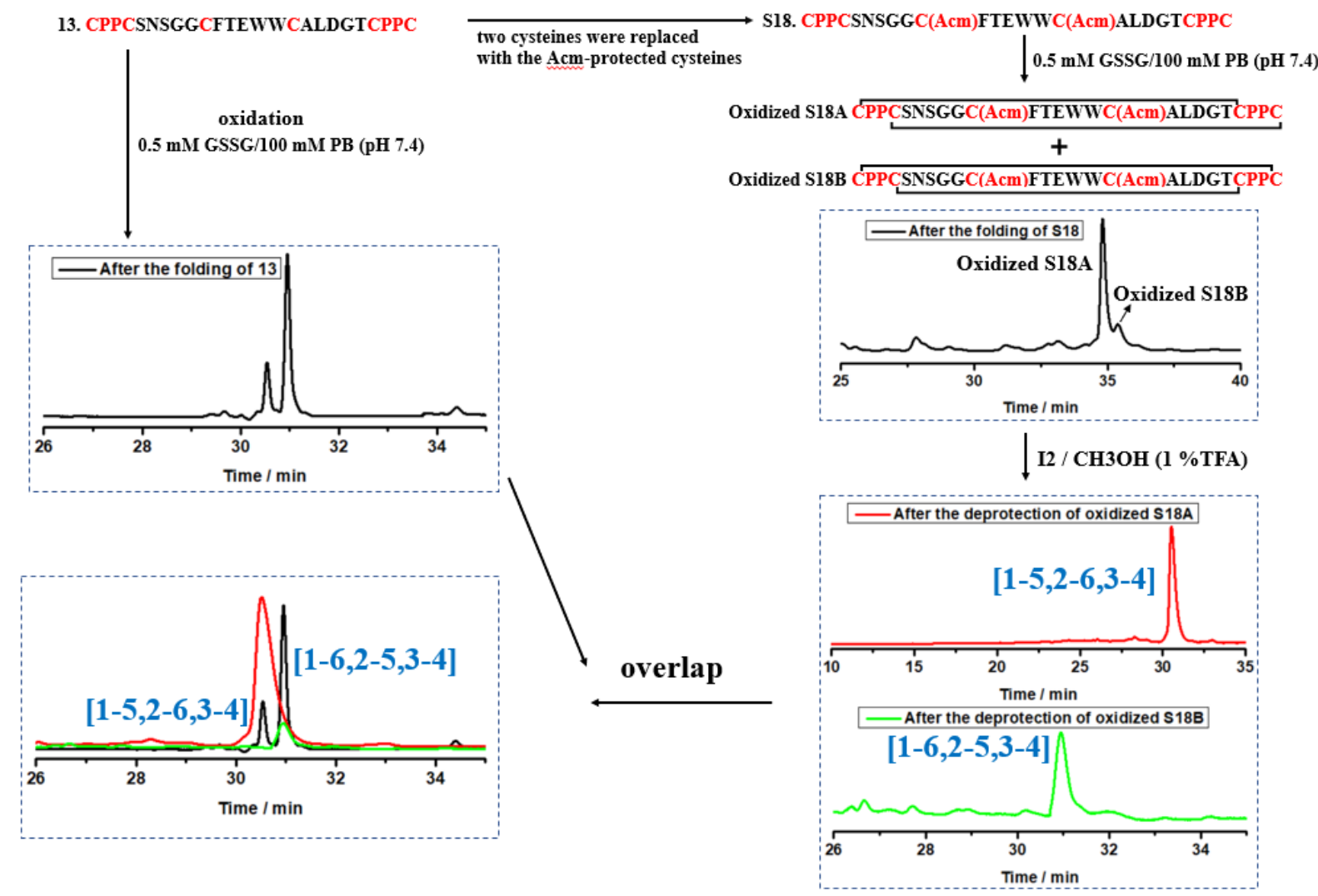

Fig S14. The flowchart to determine the disulfide pairing of $\mathbf{1 3}$. 


\subsection{FP competition curves of the binding of peptides (oxidized 12 and 13) to MDM2}

(a) 12. CPPCHGRPTCDSFTNCWELLTCPPC

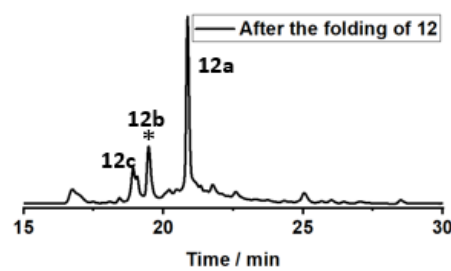

(d) 13. CPPCSNSGGCFTEWWCALDGTCPPC

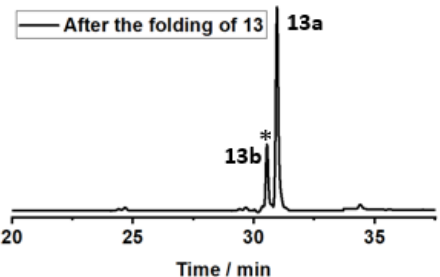

(b)

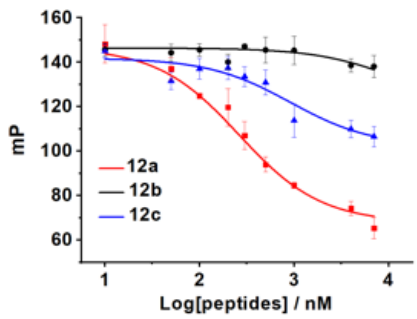

(e)

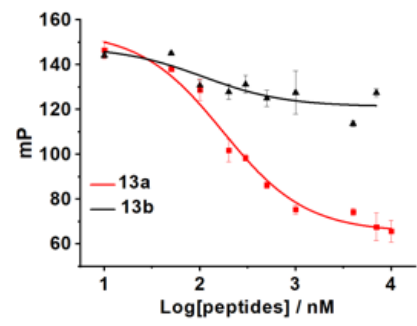

(c)

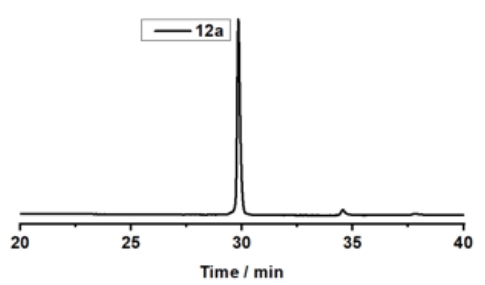

(f)

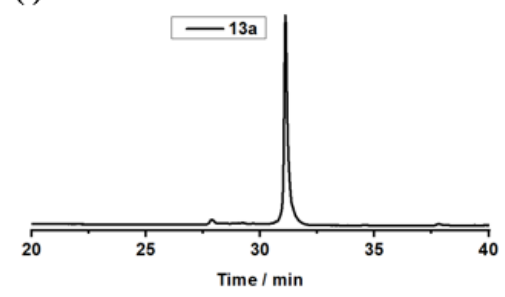

(g)
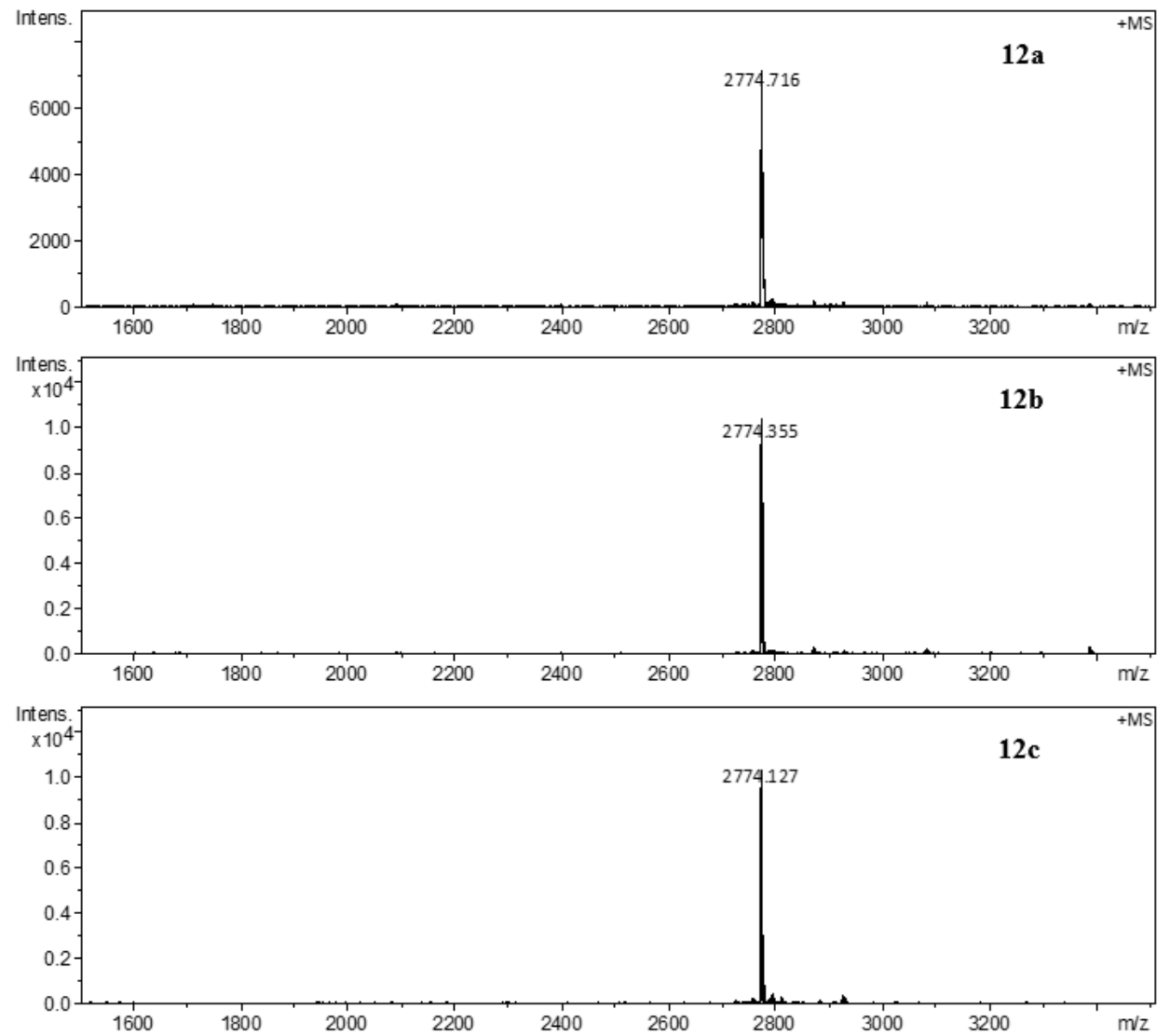


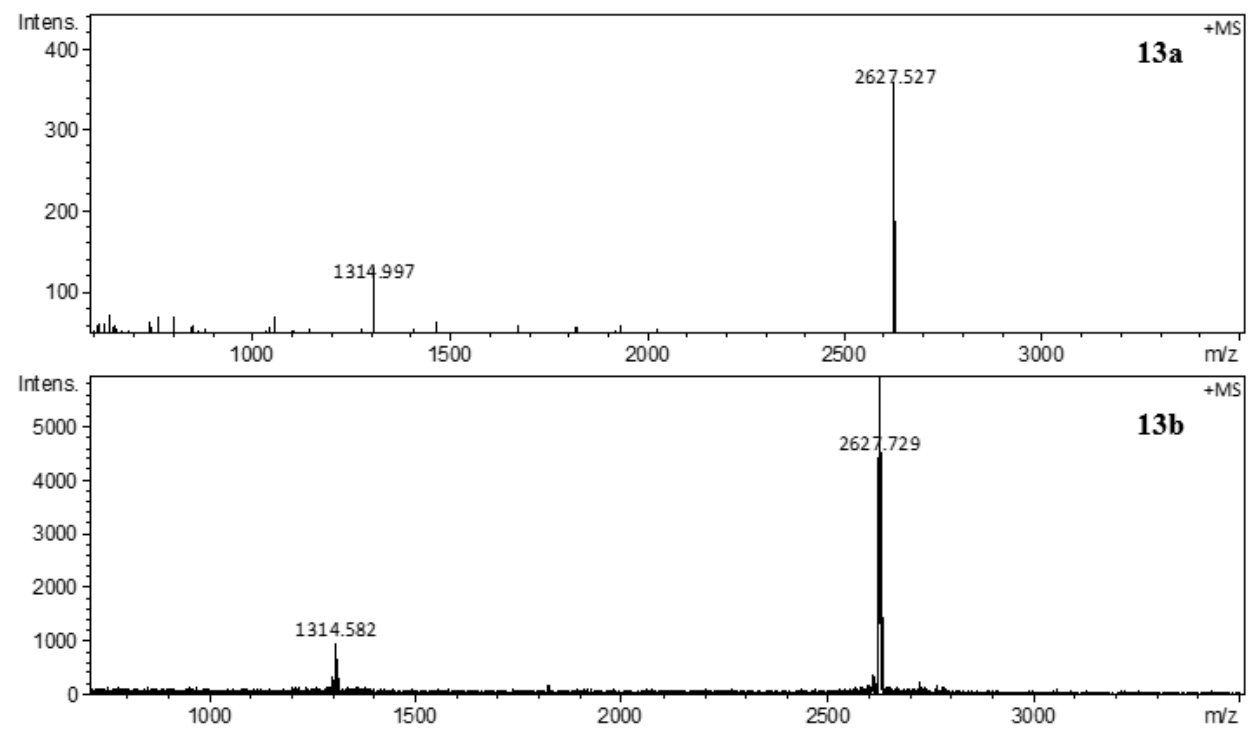

Fig S15. (a) Chromatogram showing the oxidation of 12 in phosphate buffer (100 mM, pH 7.4) containing $0.5 \mathrm{mM}$ oxidized glutathione (GSSG). (b) FP competition curves showing the binding of 12a, 12b and 12c to MDM2. (c) The 12a used for the FP assays were purified using HPLC to a purity of $>95 \%$. (d) Chromatogram showing the oxidation of 13 in phosphate buffer (100 mM, pH 7.4) containing $0.5 \mathrm{mM}$ oxidized glutathione (GSSG). (e) FP competition curves showing the binding of 13a and 13b to MDM2. (f) The 13a used for the FP assays were purified using HPLC to a purity of $>95 \%$. (g) Mass spectrum of the 12a, 12b, 12c, 13a and $13 \mathbf{b}$. 


\section{References}

1. Zheng, Y.; Zhai, L.; Zhao, Y.; \& Wu, C. Orthogonal Cysteine-Penicillamine Disulfide Pairing for Directing the Oxidative Folding of Peptides. J. Am. Chem. Soc. 2015, 137, 15094-15097.

2. Zheng, Y.; Meng, X.; Wu, Y.; Zhao, Y.; \& Wu, C. De novo design of constrained and sequence-independent peptide scaffolds with topologically-formidable disulfide connectivities. Chem. Sci. 2018, 9, 569-575.

3. Wünsch, E.; Moroder, L.; Göhring-Romani, S.; Musiol, H. J.; Göhring, W.; \& Bovermann, G. Synthesis of the bis-cystinyl-fragment 225-232/225'-232'of the human IgG1 hinge region. Int. J. Pept. Res. Ther. 1988, 32, 368-383.

4. Nikolovska-Coleska, Z.; Wang, R.; Fang, X.; Pan, H.; Tomita, Y.; Li, P.; \& Wang, S. Development and optimization of a binding assay for the XIAP BIR3 domain using fluorescence polarization. Anal. Biochem. 2004, 332, $261-273$. 